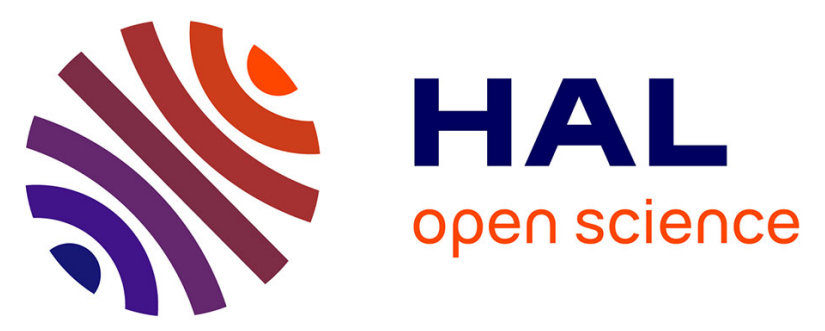

\title{
Link Between the Dynamics of Granular Flows and the Generated Seismic Signal: Insights From Laboratory Experiments
}

Maxime Farin, Anne Mangeney, Julien De rosny, Renaud Toussaint, Phuong-Thu Trinh

\section{To cite this version:}

Maxime Farin, Anne Mangeney, Julien De rosny, Renaud Toussaint, Phuong-Thu Trinh. Link Between the Dynamics of Granular Flows and the Generated Seismic Signal: Insights From Laboratory Experiments. Journal of Geophysical Research: Earth Surface, 2018, 123 (6), pp.1407-1429. 10.1029/2017JF004296 . hal-01907646

\section{HAL Id: hal-01907646 \\ https://hal.science/hal-01907646}

Submitted on 29 Oct 2018

HAL is a multi-disciplinary open access archive for the deposit and dissemination of scientific research documents, whether they are published or not. The documents may come from teaching and research institutions in France or abroad, or from public or private research centers.
L'archive ouverte pluridisciplinaire HAL, est destinée au dépôt et à la diffusion de documents scientifiques de niveau recherche, publiés ou non, émanant des établissements d'enseignement et de recherche français ou étrangers, des laboratoires publics ou privés. 


\section{Journal of Geophysical Research: Earth Surface}

\section{RESEARCH ARTICLE \\ 10.1029/2017JF004296 \\ Key Points: \\ Link Between the Dynamics of Granular Flows and the Generated Seismic Signal: Insights From Laboratory Experiments}

-We compare the dynamics and the generated seismic signal of experimental granular flows on a rough plane inclined at various angles

- Granular flow seismic efficiency decreases as the slope angle increases and is about 90 times lower than for the impact of a single grain

- The shape of the seismic signal changes after a critical slope angle because the flow regime changes from a dense to a more agitated flow

Correspondence to:

M. Farin,

maxime.farin@espci.fr

Citation:

Farin, M., Mangeney, A., de Rosny, J., Toussaint, R., \& Trinh, P.-T. (2018). Link between the dynamics of granular flows and the generated seismic signal: Insights from laboratory experiments. Journal of Geophysical Research Earth Surface, 123, 1407-1429. https://doi.org/10.1029/2017JF004296

Received 24 MAR 2017 Accepted 19 APR 2018 Accepted article online 11 MAY 2018 Published online 21 JUN 2018

○2018. American Geophysical Union All Rights Reserved.

\author{
Maxime Farin ${ }^{1}$ iD, Anne Mangeney ${ }^{2,3}$, Julien de Rosny ${ }^{1}$, Renaud Toussaint ${ }^{4}$ (D), \\ and Phuong-Thu Trinh ${ }^{5}$ \\ ${ }^{1}$ Institut Langevin, ESPCI Paris, PSL Research University, CNRS, Paris, France, ${ }^{2}$ Institut de Physique du Globe de Paris, \\ Sorbonne Paris Cité, CNRS (UMR 7154), Paris, France, ${ }^{3}$ ANGE team, CEREMA, Inria, Lab. J.-L. Lions, CNRS, Paris, France, \\ ${ }^{4}$ Institut de Physique du Globe de Strasbourg/EOST, CNRS, Strasbourg, France, ${ }^{5}$ Total E\&P/SEISCOPE/university of \\ Grenoble Alpes
}

Abstract Granular column collapse experiments have been conducted on a flat rough surface tilted at various angles with synchronous measurements of the flow dynamics and the emitted seismic signal. Our results show that the ratio of radiated seismic energy to potential energy lost by the granular flows decreases slightly from $0.033 \%$ to $0.017 \%$ with increasing slope angle on a poly(methyl methacrylate) (acrylic) plate. This is about 90 times lower than for the impact of a single particle of the same diameter. The experimental granular flows generated signals with frequencies lower than $20 \mathrm{kHz}$, with a mean value around $5 \mathrm{kHz}$, which are shown to be similar to the frequencies emitted by a single-particle impact. The rise phase and maxima of the amplitude and frequencies of the seismic signals generated by our experimental granular flows are mostly controlled by flow motion in the direction normal to the slope, while their decay phase depends on downslope particle speeds. The granular flow regime changes from dense to more agitated flows above a critical slope angle that is about half the friction angle of the granular material. This change is reflected in (1) the shape of the temporal variation of the seismic amplitude and frequencies, with a decay phase lasting much longer and (2) the shape of the cumulative radiated seismic energy, which changes above the same critical slope angle. Implications of these results for the interpretation of seismic emissions from experimental and natural granular flows are discussed.

\section{Introduction}

Over the last two decades, many studies have attempted to obtain insights into the dynamics of gravitational instabilities (e.g., avalanches, landslides, rockfalls, and debris flows) using the seismic signals generated from these events (e.g., Arattano, 2000; Brodsky et al., 2003; Dammeier et al., 2011; Favreau et al., 2010; Hibert, Ekström, et al., 2014; Huang et al., 2007; Kanamori \& Given, 1982; Kean et al., 2015; Moretti et al., 2015; Pérez-Guillén et al., 2016; Suriñach et al., 2005; Yamada et al., 2016; Zhao et al., 2015). Defining seismic attributes that can distinguish gravitational flows from other sources, like volcano-tectonic earthquakes, is crucial for hazard assessment and monitoring in volcanic or seismic environments. This, however, requires an understanding and quantification of the processes at the origin of the generated seismic signal. The high-frequency $(>1 \mathrm{~Hz})$ seismic signals recorded at some distance from landslides have a typical cigar shape with an emergent arrival (Dammeier et al., 2011; Hibert et al., 2011; Hibert, Mangeney, et al., 2014; Moretti et al., 2012; Pérez-Guillén et al., 2016; Schneider et al., 2010). Different interpretations have been proposed for this characteristic shape. Suriñach et al. (2005) suggested that it can be due to the fact that the mass moves forward, then away from the seismic station, because the amplitude of the emitted signal decays rapidly with distance. In addition, Schneider et al. (2010) and Hibert et al. (2011) observed that this shape is visible on seismic recordings at different distances from the event, which suggests that the amplitude of the signal is directly related to the dynamics of the flow. Hibert, Ekström, et al. $(2014,2017)$ found that the normalized envelope of the high-frequency seismic signal generated by several large landslides is very similar to the temporal evolution of the modulus of the normalized bulk momentum inferred from inversion of the long-period seismic waves. In addition, the maximum of the seismic envelope was observed to be proportional to the maximum bulk momentum (Hibert, Ekström, et al., 2017). Lévy et al. (2015) found similarities between 
the time variation of the seismic energy and that of the force simulated for rockfalls over complex topography in Montserrat. Finally, the complex topography of the ground has been shown to also affect the signal amplitude and shape (Favreau et al., 2010; Lévy et al., 2015; Yamada et al., 2013). Nevertheless, the seismic signal generated by gravitational events depends on numerous factors, such as particle diameter (Farin et al., 2015; Huang et al., 2004), the presence of abrupt changes in slope angle and topographic irregularities in the bed (Allstadt, 2013; Favreau et al., 2010; Moretti et al., 2015), the presence of an erodible bed (Bachelet et al., 2018; Kean et al., 2015), and propagation of seismic waves in complex heterogeneous media (Aki \& Richards, 1980). However, given the lack of direct visual observations of the dynamics of natural granular flows, it has not yet been possible to relate the amplitude of the seismic signal generated by a gravitational event to its dynamic parameters such as its speed or potential energy lost.

Parallel to field investigations, many scaled laboratory experiments of granular flows have been conducted since the 1980s to better understand the dynamics and deposition of landslides (e.g., see reviews; Andreotti et al., 2013; Delannay et al., 2017; GdR Midi, 2004). These experiments include horizontal axisymmetrical granular column collapses (e.g., Lajeunesse et al., 2004; Lube et al., 2004; Roche et al., 2011) and 2-D granular collapses in horizontal (Balmforth \& Kerswell, 2005; Lacaze \& Kerswell, 2009; Lube et al., 2005; Roche et al., 2008, 2013; Siavoshi \& Kudrolli, 2005) or inclined flat channels (Dufresne, 2012; Farin et al., 2014; Hogg, 2007; Huang et al., 2004; Lube et al., 2011; Mangeney et al., 2010; Sulpizio et al., 2016). In particular, Mangeney et al. (2010) and Farin et al. (2014) showed the existence of a critical slope angle above which the dynamics of granular flows change dramatically. This change is related to the development of a slow propagation phase at the end of the deceleration phase of the flow front, which significantly increases the duration and the runout distance of the flow front as the slope angle approaches the friction angle of the granular material. A key question is whether the seismic signal generated by the flow reflects the change in its dynamics and in particular the different regimes experienced by the flowing material. If so, analysis of the signal may provide a key tool to detect these changes and also to better quantify them and understand their origin.

Recent laboratory studies have used vibration sensors to monitor seismic and force signals generated by granular flows. For example, Huang et al. (2004) used a piezoelectric hydrophone to show that the mean frequency of the signal of a granular flow decreases when the particle diameter increases. More recently, Hsu et al. (2014) characterized the statistic distribution of fluctuating forces at the base of both dry and saturated granular flows in a rotating drum using force sensors. Farin et al. (2015) and Bachelet et al. (2018) have shown that it is possible to retrieve the mass and speed of individual impactors on various substrates (smooth, rough, or erodible) from the generated seismic signal. However, to our knowledge, no laboratory study has attempted to relate granular flow dynamics to the associated seismic emissions. The advantage of this approach is to control individually all of the granular flow parameters that can have an influence on the generated seismic signal (e.g., particle diameter, released granular volume, bed topography, rough or erodible bed, and elastic properties of the propagating media) to better understand the effect of each of these parameters. In this paper, we conduct granular column collapse experiments on a flat, rough plate that is inclined at various angles. Piezoelectric accelerometers are fixed below the plate and record the seismic signal generated by the granular flows. As a starting point, the only parameter varied in these experiments is the slope angle of the flat plate with respect to the horizontal. A uniformly flat slope is somewhat unrealistic in nature, where the slope angle changes abruptly or smoothly, possibly affecting the runout distance, the flow dynamics (e.g., Jessop et al., 2012; Sulpizio et al., 2016) and therefore the generated seismic signal (e.g., Favreau et al., 2010; Lévy et al., 2015). For example, Sulpizio et al. (2016) reported that the ratio of the average slope in the depositional area to that of the upstream channel ranged from 0.26 to 0.45 for hot avalanches at Mount Vesuvius, Italy. They showed experimentally that stronger changes of slope angles (lower ratios) cause shorter runout distances. However, here we would like to separate the effect of flow dynamics from that of complex bed topography (i.e., changes of slope and flow direction) on the generated seismic signal. Consequently, we conduct experiments on planar surfaces having an uniform slope. Synchronous video recording of the granular flow and measurement of the emitted seismic signal allows us to relate the seismic parameters (amplitude, radiated seismic energy, and frequency) to the flow dynamics. With this simple experimental setup, our primary objective is to answer the following questions: (1) What proportion of the potential energy lost by the granular flow is converted into radiated seismic energy (defined here as the seismic efficiency) and does this amount depend on time or slope angle? (2) How do the seismic efficiencies and frequency content of the signals generated by the experimental granular flows compare with those of natural rockfalls? (3) How do they compare with those of a single-particle impact? (4) Are the amplitude and frequency of the seismic 


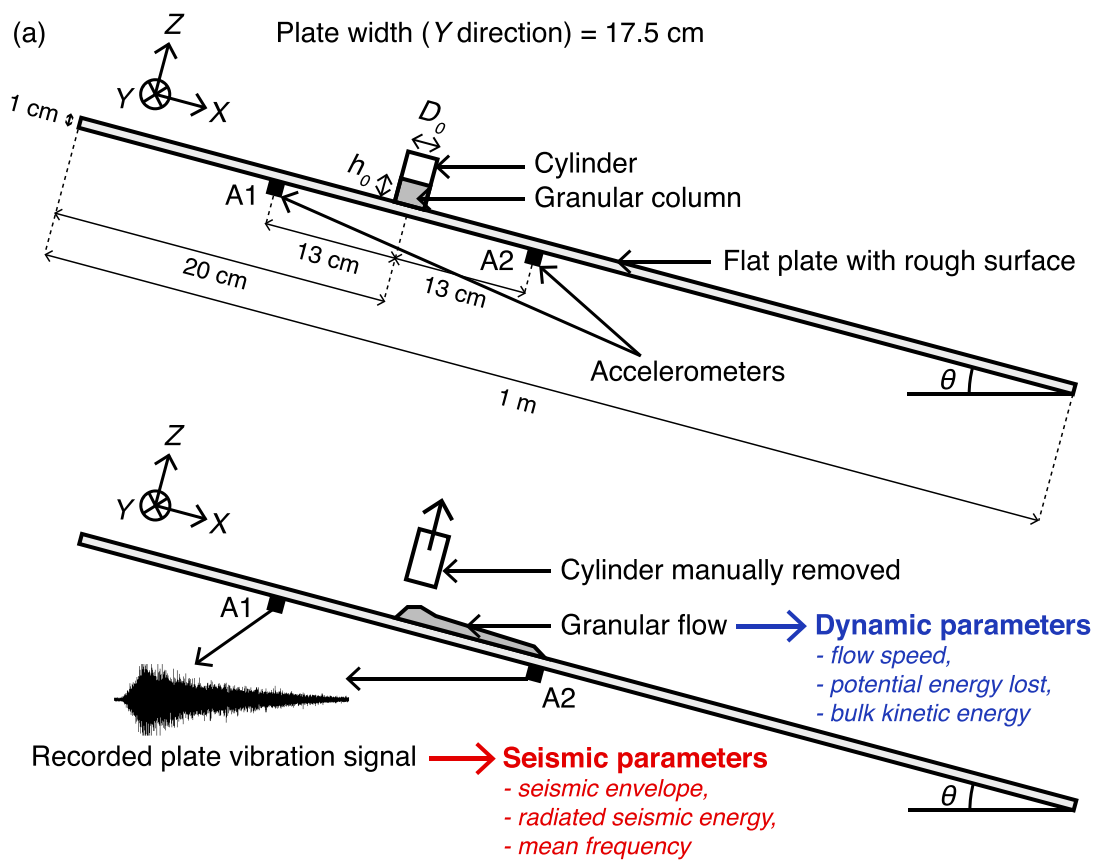

(b)

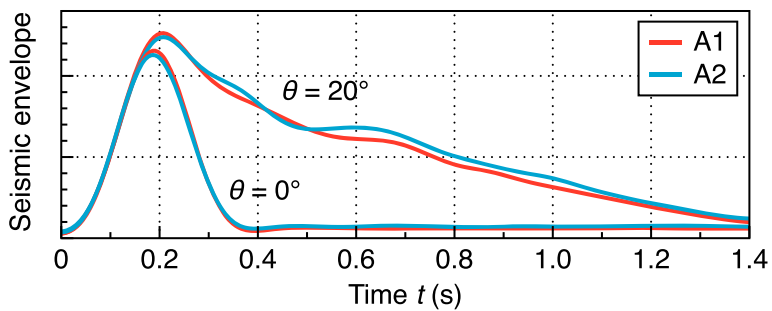

Figure 1. (a) Schematic of the experimental setup (to scale). The granular column of height $h_{0}=2.36 \mathrm{~cm}$ and diameter $D_{0}=2.95 \mathrm{~cm}$ is initially contained in a cylinder placed on a flat, rough PMMA plate of thickness $h_{p}=1 \mathrm{~cm}$, inclined at slope angle $\theta$. Two accelerometers, $A 1$ and $A 2$, are fixed below the plate. When the cylinder is removed, the granular column collapses down the slope and the granular flow generates a plate vibration, which is recorded by the accelerometers. (b) Envelope of the seismic signal measured by the two accelerometers for a granular collapse at slope angles $\theta=0^{\circ}$ and $\theta=20^{\circ}$. The amplitude envelope does not depend on the position of the measurement, therefore, the wave field can be considered as diffuse. PMMA = poly(methyl methacrylate).

signal controlled by the speed of the center of mass of the granular flow or by the speed of the flow front? (5) Does the seismic signal reveal any dramatic change in the dynamic regime of granular flows when the slope angle is increased?

After a brief description of the experimental setup in section 2, the dynamic and seismic parameters used in this work are introduced in section 3. The evolution of the seismic signal and its link with granular flow dynamics when the slope angle is increased are discussed in section 4. In section 5, we compare our experimental results to seismic observations of natural rockfalls and landslides.

\section{Experimental Setup and Preliminary Observations}

A cylindrical granular column is released on a flat poly(methyl methacrylate), PMMA, plate of thickness $h_{p}=1 \mathrm{~cm}$, which is inclined at different constant angles $\theta=0^{\circ}, 5^{\circ}, 10^{\circ}, 15^{\circ}$, and $20^{\circ}$ with respect to the horizontal (Figure 1a). The column has a mass $M \simeq 77.4 \mathrm{~g}$, initial height $h_{0}=2.36 \mathrm{~cm}$, and initial diameter $D_{0}=2.95 \mathrm{~cm}$ (i.e., an aspect ratio $a=h_{0} / D_{0}=0.8$ ). The granular mass is composed of steel beads of density $\rho_{s}=7,800 \mathrm{~kg} / \mathrm{m}^{3}$ and diameter $d=2 \mathrm{~mm}$. The PMMA plate is made rough by gluing a layer of the same beads on its surface. The repose friction angle $\delta$ of the beads on this plate is estimated to be about $27^{\circ}$, by measuring the angle formed with respect to the horizontal of a pile of beads slowly poured on the rough plate, just after it collapses. 


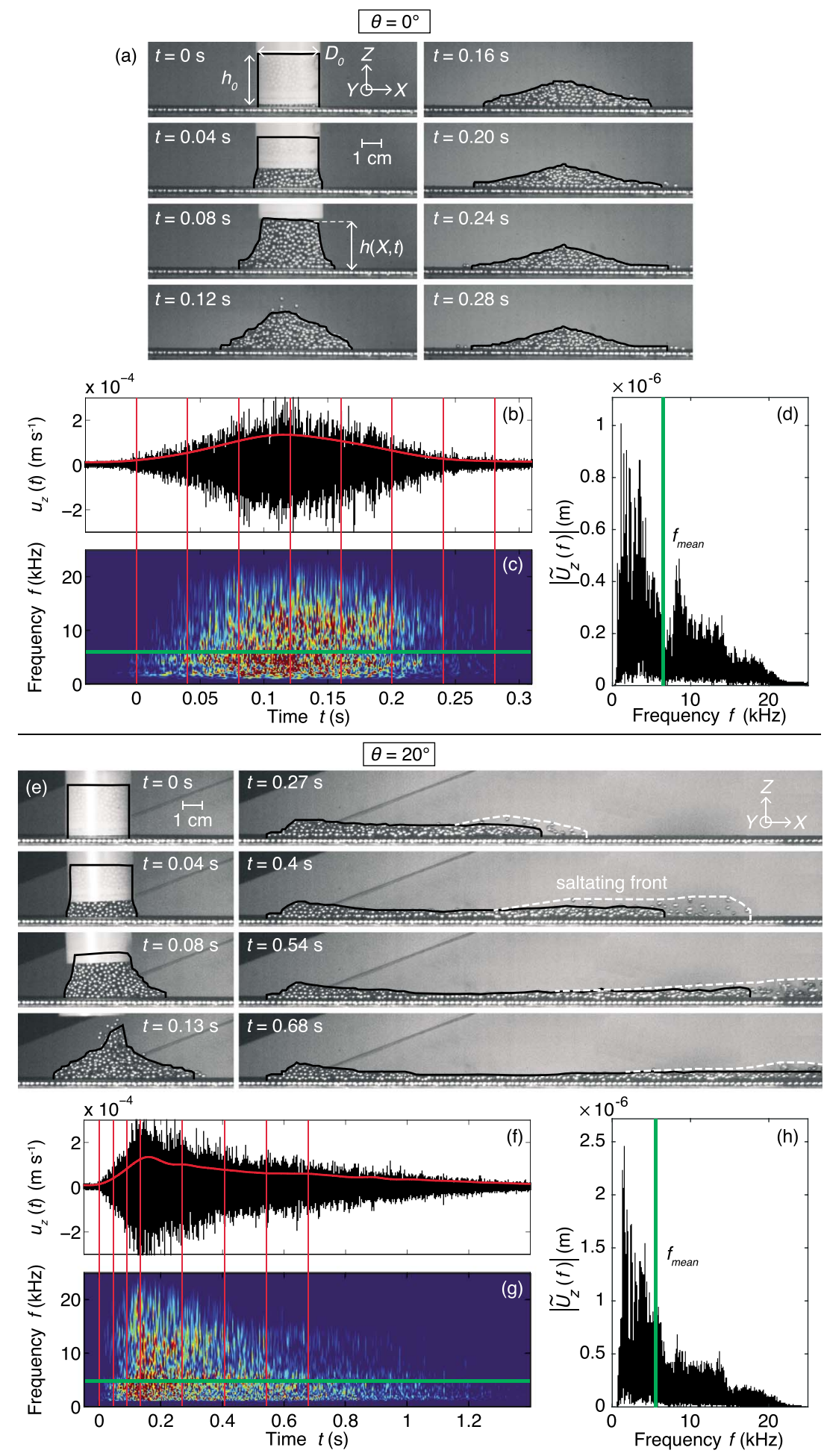

Figure 2. (a and e) Images at successive moments in time $t$ of the granular collapse of a cylindrical column of mass $M=77.4 \mathrm{~g}$, initial height $h_{0}=2.36 \mathrm{~cm}$, initial diameter $D_{0}=2.95 \mathrm{~cm}$, aspect ratio $a=0.8$ and composed of steel beads of diameter $2 \mathrm{~mm}$ for slope angle (a) $\theta=0^{\circ}$ and (e) $\theta=20^{\circ}$. The contour of the consistent flow is measured from the images (black line). The white dashed line in (e) highlights the saltating front. The black and white lines superimpose when some particles are saltating along the consistent flow in the transverse $Y$ direction. (b and f) Plate velocity in the normal direction $u_{Z}(t)$ (i.e., seismic signal) generated by the granular collapse. The thick red line is the signal envelope. (c and g) Spectrogram of the seismic signal $u_{Z}(t)$ (normalized scale). The vertical red lines indicate the times the images were obtained. ( $\mathrm{d}$ and $\mathrm{h}$ ) Amplitude spectrum $\left|\tilde{U}_{Z}(f)\right|$ of the seismic signal $u_{Z}(t)$. The mean frequency $f_{\text {mean }}$ is indicated by a green line on $(c),(d),(g)$, and $(h)$. 


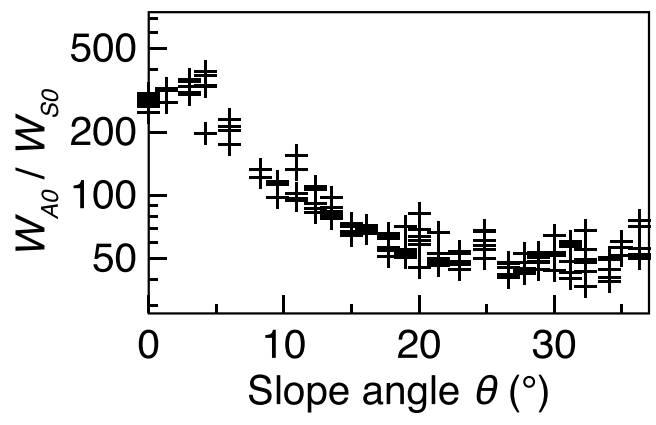

Figure 3. Ratio of the energy $W_{A 0}$ of the normal mode $A_{0}$, measured on the surface of the plate, to the energy $W_{S 0}$ of the longitudinal mode $S_{0}$, measured on the lateral side of the plate, when a particle is dropped from the same height on the plate inclined at different slope angles $\theta$ with respect to the horizontal. The energy ratio $W_{A 0} / W_{S 0}$ is the ratio of the integrals of the squared impact signals measured on the surface and on the lateral side of the plate, respectively.
The granular column is initially contained inside a cylinder and collapses when the cylinder is removed (Figure 1a). Parameters quantifying the flow dynamics (i.e., the flow speed, the potential energy lost, the kinetic energy, and the total energy lost) are determined from the video of the granular flows, recorded using a Photron SA4 camera (500 fps; Figures 2a and 2e). When the plate is horizontal (i.e., for $\theta=0^{\circ}$ ), the granular column collapse is axisymmetrical (Figure 2a). The granular flow for $\theta=20^{\circ}$ looks similar to that of the horizontal case for $t<0.13 \mathrm{~s}$ (Figure 2e). Then a thin layer of particles following a front of saltating particles propagates down the slope. The runout distance increases with the slope angle $\theta$.

The plate surface vibration acceleration $a_{z}(t)$ (i.e., the seismic signal) generated by the granular flows is measured in the direction normal to the plate using two monocomponent piezoelectric accelerometers (type 8309, Brüel \& Kjaer) with a flat response between $1 \mathrm{~Hz}$ and $54 \mathrm{kHz}$ and an acquisition rate of $500 \mathrm{kHz}$ (Figure 1a). During the granular collapse, the two sensors measure seismic signals with envelopes that have a similar shape and amplitude because the radiated seismic energy is equidistributed within the plate over a timescale much shorter than the duration of the flow propagation (Figure 1b). This is further discussed in section 3.2. The measured acceleration signals $a_{z}(t)$ are then integrated and high-pass filtered above $1,000 \mathrm{~Hz}$ (to remove the low-frequency noise resulting from signal integration) to obtain the plate normal vibration speed signals $u_{Z}(t)$ (Figures $2 \mathrm{~b}$ and $2 \mathrm{f}$ ). From the speed signal $u_{Z}(t)$, we determine the seismic parameters that are compared to the dynamic parameters in section 4 (i.e., the seismic envelope, the radiated seismic energy, and the mean frequency).

The spectrogram and amplitude spectrum $\left|\tilde{U}_{Z}(f)\right|$ of the vibration speed $u_{Z}(t)$ reveal that most of the radiated seismic energy is between $\sim 1 \mathrm{kHz}$ and $20 \mathrm{kHz}$, regardless of the slope angle $\theta$ (Figures $2 \mathrm{c}, 2 \mathrm{~d}, 2 \mathrm{~g}$, and $2 \mathrm{~h}$ ). In this frequency range, the thin PMMA plate is only excited by two fundamental modes: the flexural Lamb mode $A_{0}$ whose displacement is normal to the plane of the plate and the longitudinal mode $S_{0}$, with displacement in the plane of the plate (Farin et al., 2016; Royer \& Dieulesaint, 2000). Higher modes have a cutoff frequency higher than $20 \mathrm{kHz}$ and are thus not recorded here (Royer \& Dieulesaint, 2000). We determined the relative energy $W_{S 0}$ of the longitudinal mode $S_{0}$ (measured with a sensor on the lateral side of the plate) with respect to the energy $W_{A 0}$ of the normal mode $A_{0}$ (measured with a sensor on the surface of the plate as in Figure 1a) when a particle impacts the plate with different angles of incidence. The energy ratio $W_{A 0} / W_{S 0}$ is the ratio of the integral of the squared signals measured in the lateral and normal directions, respectively (Figure 3 ). When the angle of incidence increases from $0^{\circ}$ to $38^{\circ}$ with respect to the normal to the plate, the energy of mode $S_{0}$ represents about $0.2 \%$ to $2 \%$ of the energy of mode $A_{0}$. Therefore, in the context of our laboratory experiments, the energy carried by longitudinal waves can be considered negligible and the normal component $u_{z}(t)$ of the plate vibration speed is sufficient to determine, with a relatively good accuracy, the absolute seismic energy radiated by granular flows.

\section{Determination of the Dynamic and Seismic Parameters of the Granular Flows}

Let us first define the observables (i.e., the dynamic and seismic parameters determined in each experiment from the video recordings of the granular flows and from the generated plate vibrations $u_{z}(t)$, respectively). The results and comparisons of these parameters for different slope angles $\theta$ are presented in section 4 .

\subsection{Dynamic Parameters}

We estimate the potential energy of a granular flow from the temporal evolution of the thickness profile $h(X, Y, t)$ of the contour of the granular flow, determined from the video recording of the experiment (black contours in Figures $2 \mathrm{a}$ and $2 \mathrm{e})$. Let $\left(0, X^{\prime}, Y^{\prime}, Z^{\prime}\right)$ be the reference frame related to gravity, where $X^{\prime}$ and $Z^{\prime}$ are the horizontal and vertical directions, respectively, and $Y^{\prime}$ is the transverse direction (i.e., over the width). If $(0, X, Y, Z)$ is the reference frame of the plate (as shown in Figures $1 \mathrm{a}, 2 \mathrm{a}$, and $2 \mathrm{e})$, we have

$$
\begin{gathered}
X^{\prime}=X \cos \theta+Z \sin \theta, \\
Z^{\prime}=-X \sin \theta+Z \cos \theta, \\
Y^{\prime}=Y,
\end{gathered}
$$


where $\theta$ is the slope angle. The potential energy is obtained by integrating the bulk energy $\rho g Z^{\prime}$ over the flow volume $V$

$$
E_{p}(t)=\rho g \iiint_{V} Z^{\prime} \mathrm{d} X^{\prime} \mathrm{d} Y^{\prime} \mathrm{d} Z^{\prime}=\rho g \int_{X} \int_{Y} \int_{Z=0}^{Z=h(X, Y, t)}(-X \sin \theta+Z \cos \theta) \mathrm{d} X \mathrm{~d} Y \mathrm{~d} Z
$$

because $d X^{\prime} d Y^{\prime} d Z^{\prime}=d X d Y d Z$. Then, by integrating over $Z$, we obtain

$$
E_{p}(t)=\rho g\left(\frac{1}{2} \int_{X} \int_{Y} h(X, Y, t)^{2} \cos \theta \mathrm{d} X \mathrm{~d} Y-\int_{X} \int_{Y} h(X, Y, t) X \sin \theta \mathrm{d} X \mathrm{~d} Y\right) .
$$

Since we do not have any image of the flow in the transverse direction $Y$, the determination of the potential energy is challenging. When the plate is horizontal, $E_{p}(t)$ can be easily determined using cylindrical coordinates because the collapse is axisymmetrical. However, for slope angles $\theta>0^{\circ}$, we assume that the thickness $h(X, Y, t)$ decreases linearly from the center line of the flow $Y=0$ in the $Y$ direction, over a constant width $W$. We adjust the value of the width $W$ by equating the energy $E_{p}(t)$ computed using cylindrical coordinates at the horizontal with that computed with a linearly decreasing thickness over $W$ and obtain $W=1.5 D_{0}$. This value is assumed to stay the same for $\theta>0^{\circ}$ although it probably decreases as the flow elongates. We may therefore slightly overestimate the potential energy for high slope angles $\theta$. Finally,

$$
E_{p}(t) \simeq \frac{1.5}{2} \rho g D_{0}\left(\frac{1}{2} \int_{X} h(X, t)^{2} \cos \theta \mathrm{d} X-\int_{X} h(X, t) X \sin \theta \mathrm{d} X\right) .
$$

The potential energy lost during the collapse is then given by

$$
\Delta E_{p}(t)=E_{p}(t=0)-E_{p}(t)
$$

The density $\rho$ of the granular flow is assumed constant and equal to $M /\left(\pi D_{0}^{2} h_{0} / 4\right) \simeq 4,800 \mathrm{~kg} / \mathrm{m}^{3}$ during the collapse, which is a crude approximation given that the front of the flow is clearly less dense than the rear at the end of the propagation (e.g., for $t \geq 0.27 \mathrm{~s}$, Figure 2e).

The successive coordinates $\left(X^{\mathrm{COM}}(t), Z^{\mathrm{COM}}(t)\right)$ at time $t$ of the center of mass of the flow in the plane $(X, Y=0, Z)$ are estimated from the contour profiles of the granular flows estimated from the video recordings (Figure 4a). At a given time $t$, the coordinate $X^{\mathrm{COM}}(t)$ is the average of the thicknesses $h\left(X_{i}, t\right)$ of the flow contour in the $X$ direction, weighted by the positions $X_{i}$ (Figure $4 b$ )

$$
X^{\mathrm{COM}}(t)=\frac{\sum_{i} h\left(X_{i}, t\right) X_{i}}{\sum_{i} X_{i}}
$$

$X^{\mathrm{COM}}(t)$ does not vary for the horizontal case (i.e., for $\theta=0^{\circ}$ ) because the granular collapse is axisymmetrical. Similarly, the coordinate $Z^{\mathrm{COM}}(t)$ is the weighted average along the $Z$ direction of the lengths $I\left(Z_{i}, t\right)$ of the flow contour in the $Z$ direction at time $t$ (Figure 4c)

$$
Z^{\mathrm{COM}}(t)=\frac{\sum_{i} l\left(Z_{i}, t\right) Z_{i}}{\sum_{i} Z_{i}}
$$

The time derivatives of the coordinates $X^{\mathrm{COM}}(t)$ and $Z^{\mathrm{COM}}(t)$ give the speeds $V_{X}^{\mathrm{COM}}(t)$ (in the $X$ direction, down the slope) and $V_{Z}^{\mathrm{COM}}(t)$ (in the $Z$ direction, toward the plate) of the center of mass, respectively. Then, the bulk kinetic energy of the granular flow is given by

$$
E_{c}(t)=\frac{1}{2} M\left(V_{x}^{\mathrm{COM}}(t)^{2}+V_{Z}^{\mathrm{COM}}(t)^{2}\right)
$$

We also picked the successive coordinates of the front of the granular flow contours $X^{\text {front }}(t)$ and of their summit (i.e., maximum flow height) $Z^{\text {summit }}(t)$ (Figure 4) and differentiated these coordinates over time to obtain the speed of the flow front $V_{\text {front }}(t)$ and the speed of the summit $V_{\text {summit }}(t)$. The front speed $V_{\text {front }}(t)$ is generally 2 to 3 times higher than the speed $V_{X}^{\mathrm{COM}}(t)$ of the center of mass (see section 4). 

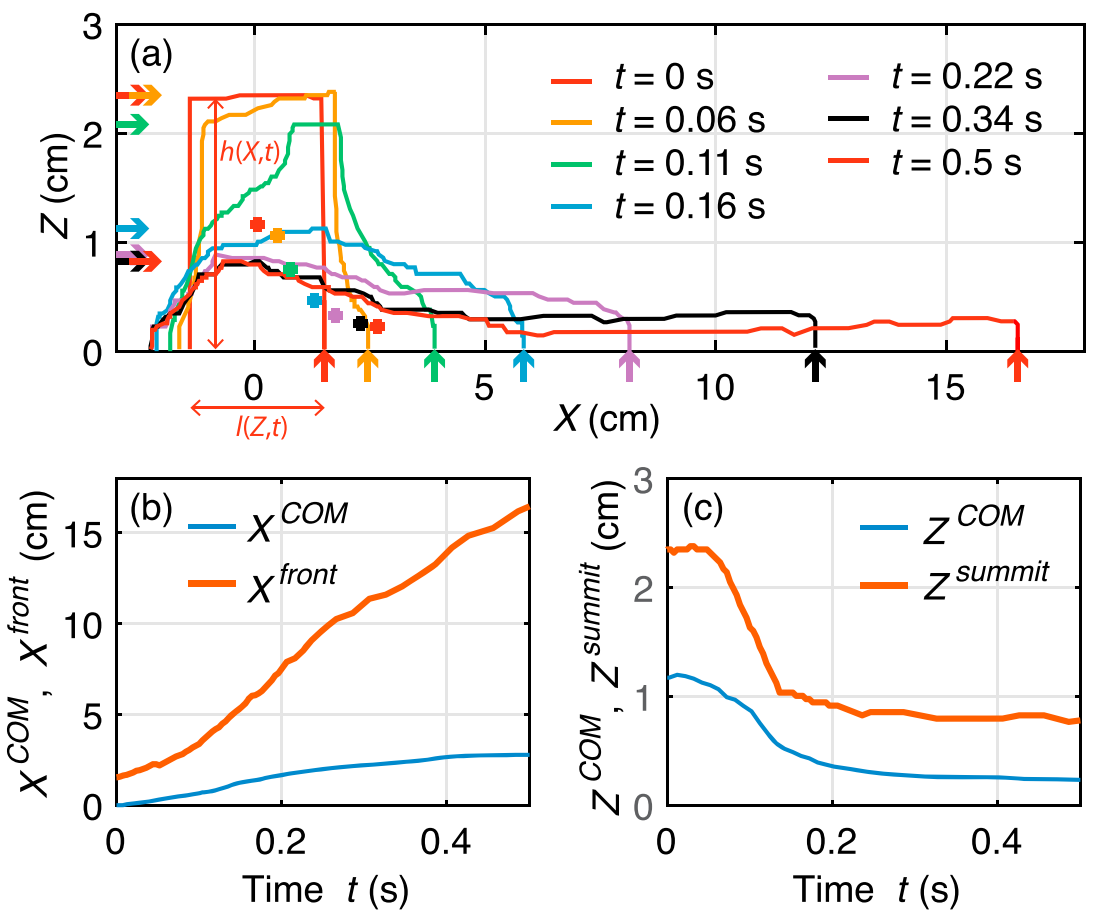

Figure 4. (a) Successive contour profiles at different times $t$ (different colors) of the granular collapse at slope angle $\theta=15^{\circ}$. The coordinates $X^{\mathrm{COM}}(t)$ and $Z^{\mathrm{COM}}(t)$ of the flow center of mass along the $X$ and $Z$ directions are determined from the weighted integration of the thicknesses $h(X, t)$ and lengths $I(Z, t)$ of the contours at times $t$, respectively (Equations (8) and (9). The dots indicate the deduced successive positions of the center of mass as a function of time. The arrows indicate the successive positions of the summit of the column $Z^{\text {summit }}(t)$ (horizontal arrows) and of the front of the granular flow $X^{\text {front }}$ (vertical arrows). (b) Coordinates $X^{\mathrm{COM}}$ of the center of mass and $X^{\text {front }}$ of the flow front in the $X$ direction. (c) Coordinates $Z^{\mathrm{COM}}$ of the center of mass and $Z^{\text {summit }}$ of the summit of the granular flow in the $Z$ direction at slope angle $\theta=15^{\circ}$.

\subsection{Seismic Parameters}

We characterize the seismic signal $u_{z}(t)$ generated by a granular flow by its envelope Env $(t)$, which is computed using the Hilbert transform of $u_{z}(t)$, as described by equation (2) of Hibert et al. (2011), (Figures 2b and $2 \mathrm{f}$ ) and by its duration $t_{s}$, which we define as the time interval for which the amplitude envelope $\operatorname{Env}(t)$ is higher than twice the average absolute noise amplitude. In the frequency domain, we define the mean frequency $f_{\text {mean }}$ by

$$
f_{\text {mean }}=\frac{\int_{0}^{+\infty}\left|\tilde{U}_{Z}(f)\right| f \mathrm{~d} f}{\int_{0}^{+\infty}\left|\tilde{U}_{Z}(f)\right| \mathrm{d} f},
$$

where $\left|\tilde{U}_{Z}(f)\right|$ is the absolute value of the time Fourier transform of the vibration speed $u_{Z}(t)$.

We also determine the amount of lost potential energy $\Delta E_{p}$ that is radiated in the form of seismic waves, as has been done for natural rockfalls and landslides (Deparis et al., 2008; Hibert et al., 2011; Hibert, Malet, et al., 2017; Lévy et al., 2015; Vilajosana et al., 2008). It is important to determine the radiated seismic energy $W_{\mathrm{el}}$ because it is often the only energy dissipated by the granular flow that can be measured in the field. The usual technique to determine the seismic energy $W_{\mathrm{el}}$ radiated by granular flows in the field is similar to that used for earthquakes in seismology (e.g., Crampin, 1965; Lancieri et al., 2012). It consists of assuming that the granular flow is a point source and integrating the energy flux measured at the seismic station over a cylinder of diameter equal to the distance between the granular flow and the station. However, in the laboratory, we cannot assume that the granular flows are point sources of seismicity. Indeed, the seismic waves emitted in the thin plate by the impacts of the particles constituting the flow are reflected many times off the lateral sides of the plate. In addition to the first wave arrival from the impacts, these reflections are also recorded by the accelerometers, which could lead to an overestimation of $W_{\mathrm{el}}$. It is possible to discard these reflections for a single impact provided that the plate is sufficiently 
large (Farin et al., 2016). However, in the current investigation it is impossible to discern each impact in the granular collapse and some impacts occur simultaneously. In fact, the seismic energy radiated by the granular flow is rapidly equidistributed within the plate. This is demonstrated by the virtually identical envelopes of the seismic signal measured at two locations on the plate (Figure 1b). This situation is sometimes referred to as a diffuse field (Farin et al., 2016; Weaver, 1985).

A method is presented here to roughly estimate the seismic energy $W_{\mathrm{el}}$ radiated by a granular flow on a thin plate in the laboratory, under diffuse field conditions. We make the strong assumption that the signal of the granular flow is composed of a large number of uncorrelated impulses generated by the impacts of the particles on the plate. If this assumption is true, then the seismic energy $W_{\mathrm{el}}$ radiated by a granular flow is the sum of the energies $W_{i}$ of the individual impacts over the signal duration $t_{s}$. This assumption was previously used by Tsai et al. (2012) to determine the seismic power generated by the bedload in rivers. The problem of estimating the seismic energy $W_{\mathrm{el}}$ radiated during a granular flow is then simplified to the estimation of the seismic energy $W_{i}$ radiated during a single impact on a thin plate in a diffuse field. A technique derived from classical methods used in room acoustics (e.g., Royer \& Dieulesaint, 2000) was adapted by Farin et al. (2016) to determine this energy from the plate vibration speed $u_{z}(t)$ emitted by the impact. According to this method, the seismic energy $W_{i}$ radiated during the impact is given by

$$
W_{i} \approx \rho_{p} v_{p} \overline{u_{z i}^{2}}
$$

where $\rho_{p}$ and $V_{p}$ are the density and volume of the plate, respectively. The parameter $\overline{u_{z i}{ }^{2}}$ is the amplitude of the squared surface vibration speed at the instant $t_{i}$ of the impact, which can be determined on the basis of the observation that, in a diffuse field, the amplitude of the envelope of the squared signal $\overline{u_{z}(t)^{2}}$ generated by an impact decreases exponentially in time with a characteristic time $\tau$ (Farin et al., 2016)

$$
\overline{u_{Z}(t)^{2}} \approx \overline{u_{Z i}{ }^{2}} \exp \left(-\frac{t-t_{i}}{\tau}\right) .
$$

The time $\tau$ of energy dissipation is a characteristic of the anelasticity of the plate material and depends on the signal frequency $f$ (Farin et al., 2016). It can be shown that the seismic energy $W_{\mathrm{el}}(t)$ radiated up to time $t$ by the granular flow is given by (see Appendix A for the demonstration)

$$
W_{\mathrm{el}}(t) \approx \frac{\rho_{p} V_{p}}{\tau} \int_{0}^{t} u_{Z}\left(t^{\prime}\right)^{2} \mathrm{~d} t^{\prime},
$$

where $u_{z}(t)$ is the surface vibration speed measured by the accelerometers during the granular flow (Figures $2 \mathrm{~b}$ and $2 \mathrm{f}$ ). The total seismic energy radiated during the whole experiment is then $W_{\mathrm{el}}=W_{\mathrm{el}}\left(t_{\mathrm{s}}\right)$, where $t_{s}$ is the duration of the seismic signal.

\section{Comparison of the Seismic and Dynamic Parameters}

For each granular flow experiment, we use equation (14) to compute the average radiated seismic energy $W_{\text {el }}$ determined from the plate vibration speed $u_{z}(t)$ measured by the two accelerometers. For the characteristic time $\tau$ in equation (14), we use $\tau \approx 0.09 f^{-1 / 2}$ (in units of s) as reported by Farin et al. (2016) for a PMMA plate of the same thickness, with $f=f_{\text {mean, }}$ the mean frequency of the seismic signal. Thus, $\tau=1.2 \times 10^{-3} \pm 10^{-4} \mathrm{~s}$ in our experiments.

\subsection{Description of the Seismic Signal}

We first describe how the seismic signal generated by a granular flow changes as the slope angle $\theta$ is increased. We do not observe any clear dependence of the envelope $\operatorname{Env}(t)$ of the seismic signal on the slope angle $\theta$ during the rise phase, until about $t=0.15 \mathrm{~s}$ (Figure $5 \mathrm{a}$ ). The maximum of the amplitude envelope is also similar $\left(\sim 15 \times 10^{-5} \pm 1.4 \times 10^{-5} \mathrm{~m} / \mathrm{s}\right)$ regardless of the slope angle $\theta$ and is reached at around the same time $t \simeq 0.15-0.17 \mathrm{~s}$. After $t \simeq 0.2 \mathrm{~s}$, the curves clearly separate as the slope angle $\theta$ is increased (see Figure $5 \mathrm{a}$ ). The duration of the decay phase only slightly increases with $\theta$ when $\theta \leq 10^{\circ}$ but significantly increases for $\theta>10^{\circ}$ and even more so as $\theta$ tends toward the friction angle $\delta \simeq 27^{\circ}$.

Similar to the signal amplitude envelope, the seismic energy $W_{\mathrm{el}}$ radiated during the collapse only slightly varies with $\theta$ for $\theta \leq 10^{\circ}$, but significantly increases for $\theta>10^{\circ}$ (Figure $5 \mathrm{~b}$ ). For $\theta \leq 10^{\circ}$, about half of the seismic energy $W_{\mathrm{el}}$ is radiated during the rise phase, for $t<0.15 \mathrm{~s}$. For $\theta=15^{\circ}$ and $\theta=20^{\circ}$, more seismic energy is radiated during the decay phase ( $63 \%$ and $82 \%$, respectively). 


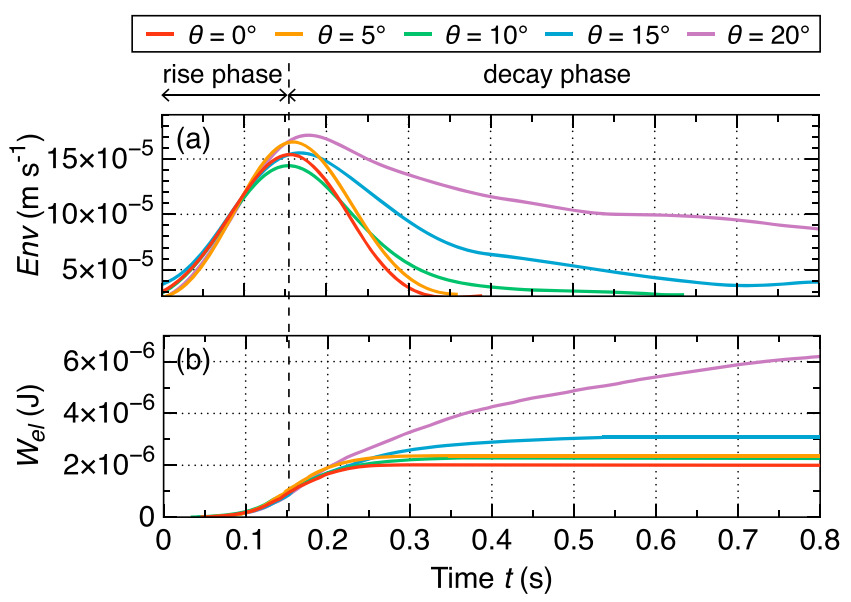

Figure 5. (a) Average envelope $\operatorname{Env}(t)$ of the measured seismic signals $u_{Z}(t)$. (b) Cumulative radiated seismic energy $W_{\mathrm{el}}(t)$ (equation (14)) of the granular flows for different slope angles $\theta$ (different colors). The vertical dashed line indicates the approximate time of the maximum of the amplitude envelopes.

\subsection{Interpretation of the Slope Angle Dependence}

As shown analytically by Tsai et al. (2012), the high-frequency seismic power radiated by the impacts of bedload particles in rivers should be directly proportional to the rate of particle impacts on the ground and to the squared impact speed. Moreover, Farin et al. (2015) showed analytically that the seismic energy $W_{\text {el }}$ radiated during a single impact is proportional to the impactor's speed to the power of $11 / 5=2.2$ on plates. Additionally, Farin (2015) reported that the energy $W_{\text {el }}$ radiated during an oblique impact decreases with the cosine of the impact angle with respect to the normal to the plate. The seismic signal generated by a granular flow should also depend on the rate, speed, and direction of the particle impacts on the bed. Therefore, in this section, we interpret the variations of the seismic signal amplitude and frequencies when the inclination angle $\theta$ of the plate increases as quantitative indications of a change of particle agitation in the flow (sometimes referred to as granular temperature in the literature, e. g., Andreotti et al., 2013; Delannay et al., 2017) or a change in flow speed and direction.

\subsubsection{Conversion of Potential and Total Energy Into Radiated Seismic Energy}

The potential energy lost by the granular flows $\Delta E_{p}(t)$ is partly converted into kinetic energy $E_{c}(t)$ and radiated into seismic waves. The potential and kinetic energy that is not converted to seismic waves is dissipated by viscous and plastic processes through binary impacts (e.g., Farin et al., 2015) and frictional contacts between the particles. Note that the total energy lost $E_{\text {tot }}(t)=\Delta E_{p}(t)+E_{c}(t)$ by the granular flows in these experiments is only slightly higher than the potential energy lost $\Delta E_{p}(t)$, because bulk kinetic energy $E_{c}(t)$ is about 1 order of magnitude lower than $\Delta E_{p}(t)$ (Figures $6 \mathrm{a}$ to $6 \mathrm{c}$ ).

The profiles of the kinetic energy $E_{c}(t)$ differ from those of the envelope $E n v(t)$ (Figures $5 \mathrm{a}$ and $6 \mathrm{~b}$ ). However, the potential energy $\Delta E_{p}(t)$ and the total energy lost $E_{\text {tot }}(t)$ exhibit a time variation similar to that of the radiated seismic energy $W_{\mathrm{el}}(t)$ during the granular collapse, with no clear dependence on the slope angle $\theta$ up to about $t=0.15 \mathrm{~s}$, followed by a stronger increase for $\theta \geq 15^{\circ}$ (Figures $5 \mathrm{~b}, 6 \mathrm{a}$, and $6 \mathrm{c}$ ). For each of the slope angles, the cumulative normalized energies $W_{\mathrm{el}}(t), \Delta E_{p}(t)$ and $E_{\mathrm{tot}}(t)$ match well (Figure $6 \mathrm{~d}$ ). The shapes of the normalized energy curves are however different for low and high slope angles $\theta \geq 15^{\circ}$, which suggests that the energy is dissipated differently, with a critical slope angle between $10^{\circ}$ and $15^{\circ}$ (i.e., around half the
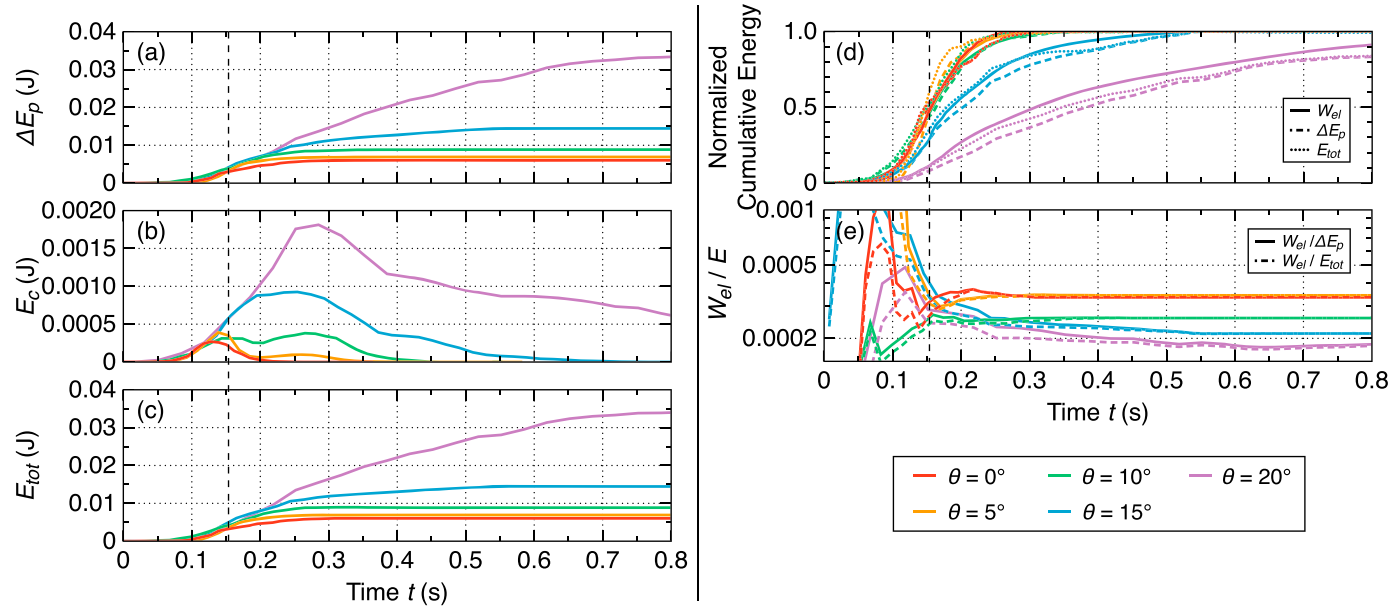

Figure 6. (a) Potential energy lost $\Delta E_{p}(t)$ (equation (7)), (b) Bulk kinetic energy $E_{c}(t)$ (equation (7)), (c) Total energy lost $E_{\text {tot }}(t)=\Delta E_{p}(t)+E_{c}(t)$, (d) Cumulative radiated seismic energy $W_{\mathrm{el}}(t)$ compared with cumulative potential energy lost $\Delta E_{p}(t)$ and total energy lost $E_{\text {tot }}(t)$, normalized by their maximum values, (e) Ratio of $W_{\text {el }}(t)$ over potential energy lost and total energy lost for the granular flows at different slope angles $\theta$ (different colors). The vertical dashed line indicates the approximate time of the maximum of the amplitude envelopes. 

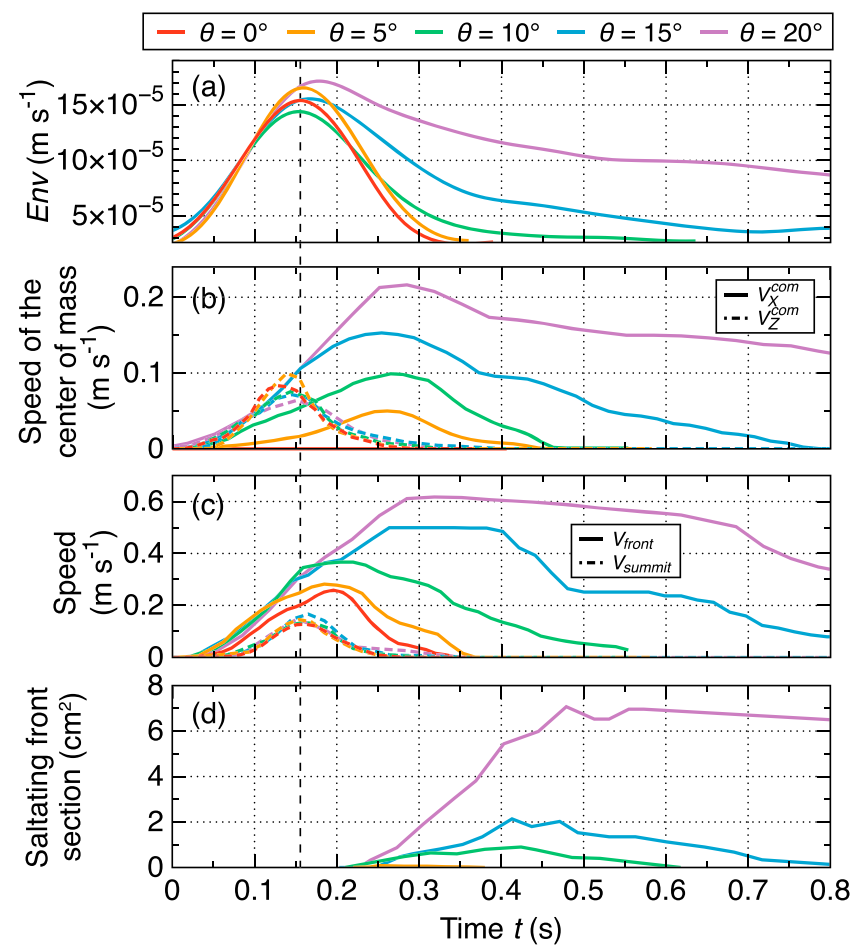

Figure 7. Comparison of (a) the seismic envelope $\operatorname{Env}(t)$ as shown in Figure $5 a$ with (b) to (d) the flow dynamics for different slope angles $\theta$ (different colors). (b) Speed of the center of mass $V_{X}^{\mathrm{COM}}(t)$ and $V_{Z}^{\mathrm{COM}}(t)$, in the $X$ and $Z$ directions, respectively. (c) Speed of the flow front $V_{\text {front }}(t)\left(X\right.$ direction) and of the column summit $V_{\text {summit }}(t)$ ( $Z$ direction). (d) Estimated cross-sectional area of the saltating front. The vertical dashed line indicates the approximate time of the maximum of the amplitude envelopes.

friction angle $\delta=27^{\circ}$ of the beads). Note that the shape of the cumulative radiated seismic energy $W_{\mathrm{el}}(t)$ matches slightly better with the cumulative total energy $E_{\text {tot }}(t)$ than with the cumulative potential energy lost $\Delta E_{p}(t)$. The same observations can be made for the radiated seismic power and the total power lost (see Appendix $B$ ). This suggests that the seismic energy radiated by granular flows should be proportional to the total dissipated energy.

The ratios of the radiated seismic energy over the potential energy lost $W_{\mathrm{el}} / \Delta E_{p}$ and over total energy lost $W_{\mathrm{el}} / E_{\mathrm{tot}}$ do not appear to be constant with time and are larger in the rise phase than in the decay phase in some experiments, for $\theta=0^{\circ}, \theta=5^{\circ}, \theta=20^{\circ}$ (Figure 6e). In these experiments, we may underestimate the potential and total energy lost when the cylinder is removed for $t<0.1 \mathrm{~s}$ because some particles are entrained upward (Figures $2 \mathrm{a}$ and 2e). Moreover, both lost and radiated energies are small in the rise phase, which leads to a larger error on the ratios. However, it is clear that the final ratios $W_{\mathrm{el}} / \Delta E_{p}$ and $W_{\mathrm{el}} / E_{\mathrm{tot}}$ decrease as the slope angle $\theta$ is increased, from $3.3 \times 10^{-4}$ for $\theta=0^{\circ}$ to $1.7 \times 10^{-4}$ for $\theta=20^{\circ}$ (Figure $6 \mathrm{e}$ ). An explanation for this observation could be that the flow potential energy is more efficiently radiated in the form of elastic waves when impacts of particles are normal to the plate at low slope angles than when impacts are more tangential to the plate for high slope angles (Bachelet et al., 2018; Farin, 2015).

\subsubsection{Interpretation of the Temporal Variation of the Signal Amplitude}

We now interpret the variation of the seismic amplitude envelope $E n v(t)$ as a function of time $t$ when the slope angle $\theta$ of the plate is increased (Figure 7a). In the rise phase, for $t<0.15 \mathrm{~s}$, the rate, speed, and direction of particle impacts on the plate, and consequently the amplitude of the seismic signal, should not be strongly affected by increasing slope angle $\theta$ because the initial driving force of the flow is controlled by the pressure gradient (Farin et al., 2014; Mangeney-Castelnau, 2003). Indeed, the pressure gradient only depends on the initial shape or height $h_{0}$ of the granular column (which is constant in these experiments) and not on the slope angle $\theta$. Until the seismic amplitude reaches its maximum for $t=0.15 \mathrm{~s}$, it seems more related to flow motion in the $Z$ direction than to motion in the $X$ direction (Figures 7a to $7 c$ ). Indeed, the speeds $V_{Z}^{\operatorname{COM}}(t)$ and $V_{\text {summit }}(t)$ in the $Z$ direction do not vary significantly as $\theta$ is increased. The same is true for the signal envelope $\operatorname{Env}(t)$. Moreover, their maxima roughly coincide at $t \simeq 0.15 \mathrm{~s}$, probably because both the rate and speed of particle 

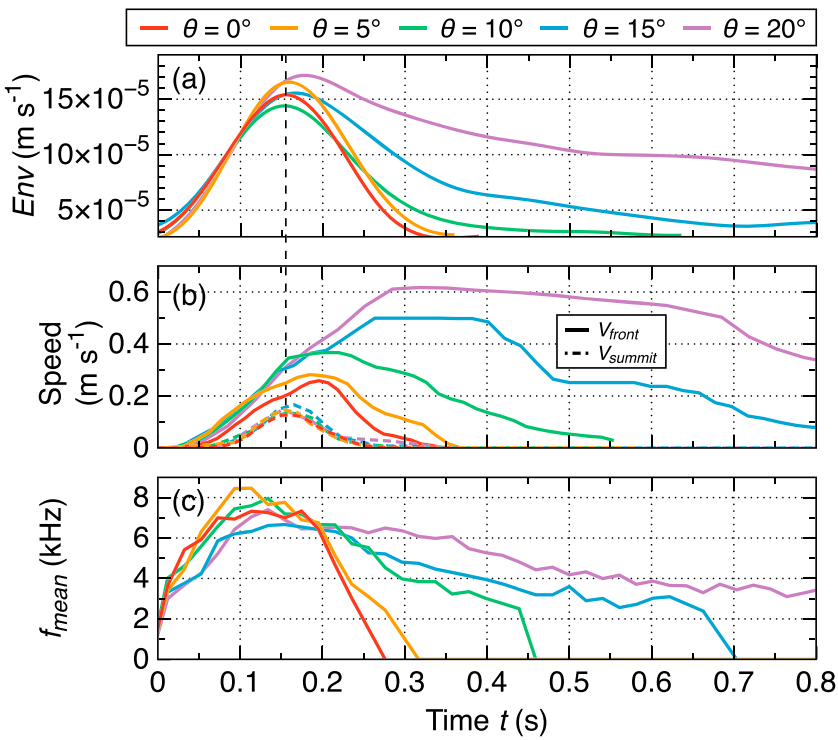

(d)

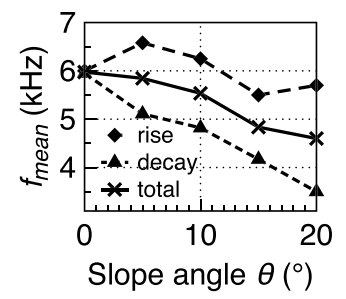

Figure 8. (a) Seismic envelope $\operatorname{Env}(t)$ as shown in Figure 5a. (b) Speed of the flow front $V_{\text {front }}(t)(X$ direction) and of the column summit $V_{\text {summit }}(t)$ ( $Z$ direction) as shown in Figure 7c. (c) Mean frequency $f_{\text {mean }}(t)$ (equation (11)) measured for successive time periods in the seismic signal, for different slope angles $\theta$ (different colors). (d) Mean frequency $f_{\text {mean }}$ computed in the rise and decay phases and for the total seismic signal as a function of the slope angle $\theta$. The vertical dashed line indicates the approximate time of the maximum of the amplitude envelopes.

impacts on the bed in the normal direction are maximized at this time. On the other hand, the maxima of the speeds $V_{X}^{\mathrm{COM}}(t)$ and $V_{\text {front }}(t)$ in the $X$ direction are reached later than those of the amplitude envelopes $\operatorname{Env}(t)$, between $t \simeq 0.2 \mathrm{~s}$ and $t \simeq 0.28 \mathrm{~s}$, and clearly increase with $\theta$ (Figures 7a to 7c).

After $t=0.15 \mathrm{~s}$, in the decay phase, the speed in the $Z$ direction decreases rapidly and the motion stops around $t=0.3 \mathrm{~s}$, regardless of $\theta$ (Figures $7 \mathrm{~b}$ and $7 \mathrm{c}$ ). Therefore, the motion in the $X$ direction has a larger influence on the seismic signal. In addition, a dilute and agitated front of 1-2 layers of saltating particles starts to develop at the flow head in the decay phase for high slope angles $\theta \geq 10^{\circ}$ (Figure 2e). Both the size and duration of the saltating front significantly increase as the slope angle $\theta$ is increased (Figure $7 \mathrm{~d}$ ). This suggests stronger particle agitation, leading to slightly higher speeds $V_{Z}$ in the $Z$ direction of the individual particles in the flow front. For example, from the videos, we measured $V_{Z} \simeq 0.19 \pm 0.05 \mathrm{~m} \mathrm{~s}^{-1}$ for $\theta=15^{\circ}$ and $V_{Z} \simeq 0.27 \pm 0.05 \mathrm{~m} \mathrm{~s}^{-1}$ for $\theta=20^{\circ}$. Because both flow speed in the $X$ direction and individual particle speed in the $Z$ direction increase in the decay phase when the slope angle $\theta$ is increased, so does the seismic amplitude (Figure 7a).

The significantly longer decay of the amplitude envelope $\operatorname{Env}(t)$ for $\theta \geq 15^{\circ}$ (Figure $7 \mathrm{a}$ ) and the change of shape of the normalized energy curves between $\theta=10^{\circ}$ and $\theta=15^{\circ}$ (Figure $6 \mathrm{~d}$ ) seem to be related to a change in the dynamics of the granular flow, from a dense granular flow toward a possibly more dilute and agitated flow with a saltating front (Figure 7d). These observations thus suggest that it is possible to detect, in the generated seismic signal, variations in granular flow dynamics when the slope angle $\theta$ is increased. Interestingly, the dynamic regime of the 2-D confined granular flow experiments conducted by Mangeney et al. (2010) and Farin et al. (2014) changes after about the same critical angle, between $\theta=10^{\circ}$ and $\theta=16^{\circ}$, with the appearance of a slow propagation phase. However, note that the regime observed here is different from a slow propagation phase, because a slow propagation phase resembles a steady uniform flow, 
Table 1

Comparison of the Ratio of the Mean Frequency $f_{\text {mean }}$ With the Ratio of Front Speed $V_{\text {front }}$ to the Power of $1 / 5$ for $\theta=15^{\circ}$ and $\theta=20^{\circ}$ and Different Times $t$

\begin{tabular}{lcc}
\hline$t(\mathrm{~s})$ & $f_{\text {mean }}\left(15^{\circ}\right) / f_{\text {mean }}\left(20^{\circ}\right)$ & {$\left[V_{\text {front }}\left(15^{\circ}\right) / V_{\text {front }}\left(20^{\circ}\right)\right]^{1 / 5}$} \\
\hline 0.45 & 0.72 & 0.92 \\
0.5 & 0.7 & 0.8 \\
0.55 & 0.75 & 0.85 \\
0.6 & 0.77 & 0.85 \\
0.65 & 0.74 & 0.84 \\
\hline
\end{tabular}

with a small and constant propagation speed, less than $25 \%$ of the maximum speed (e.g., see Figure 7 of Farin et al., 2014). In contrast, in the present experiments, the flow speed is still high in the deceleration phase and does not tend as clearly toward a constant value (Figure 7c).

\subsubsection{Variation of the Signal Mean Frequency With Slope Angle}

Similar to the signal amplitude envelope $\operatorname{Env}(t)$, the mean frequency $f_{\text {mean }}$ of the seismic signal does not seem to clearly depend on slope angle $\theta$ for $t<0.2 \mathrm{~s}$ (within the error bar of $\pm 500 \mathrm{~Hz}$ ) and decreases less rapidly during the decay phase when the slope angle $\theta$ is increased (Figures $8 \mathrm{a}$ and $8 \mathrm{c}$ ). This similarity could be explained by the fact that both the seismic amplitude and the mean frequency increase with the speed of the particles. Farin et al. (2015) showed that the mean frequency of the seismic signal generated by a single impact is inversely proportional to the duration of the impact. According to Hertz's theory of elastic impact, this duration can be written

$$
T_{c} \simeq 2.87\left(\frac{m^{2}}{R E^{* 2} V_{Z}}\right)^{1 / 5},
$$

where $m, R$, and $V_{Z}$ are the impactor's mass, radius and impact speed in the $Z$ direction, respectively, and $E^{*}$ is an elastic modulus given by $1 / E^{*}=\left(1-v_{i}^{2}\right) / E_{i}+\left(1-v_{g}^{2}\right) / E_{g}$, where $v_{i}$ and $v_{g}$ are Poisson's ratios, and $E_{i}$ and $E_{g}$ are Young's moduli for the impactor and the ground, respectively (Johnson, 1985). The mean frequency of the signal of an impact is therefore proportional to the impact speed $V_{Z}$ to the power $1 / 5$. If we assume that the particle impacts in the granular flow are uncorrelated, then the sum of the impacts should not affect the shape of the amplitude spectrum of the impact forces (Tsai et al., 2012). Consequently, the mean frequency $f_{\text {mean }}$ of the signal generated by a granular flow should also increase with particle speeds. For example, for $t$ between $0.45 \mathrm{~s}$ and $0.65 \mathrm{~s}$, when the saltating front is developed, the ratio of front speeds $V_{\text {front }}$ to the power of $1 / 5$ between $\theta=15^{\circ}$ and $\theta=20^{\circ}$ is $\sim 0.85 \pm 0.05$ and the ratio of mean frequencies $f_{\text {mean }}$ is $\sim 0.75 \pm 0.05$, which are similar (see Table 1 and Figures $8 \mathrm{~b}$ and $8 \mathrm{c}$ ). Thus, the frequency $f_{\text {mean }}$ does not significantly depend on the slope angle $\theta$ for $t<0.2 \mathrm{~s}$, probably because the particle speeds do not vary much before this time when $\theta$ is increased.

The mean frequency $f_{\text {mean }}=6 \pm 0.5 \mathrm{kHz}$ in the rise phase does not depend on the slope angle $\theta$, but it clearly decreases as $\theta$ is increased in the decay phase (Figure $8 \mathrm{~d}$ ). Indeed, the decay phase contains mostly low frequencies (typically $<5 \mathrm{kHz}$ ) and lasts longer at high slope angles (Figure $8 \mathrm{c}$ ). As a consequence, the mean frequency $f_{\text {mean }}$ measured for the total seismic signal decreases as $\theta$ is increased (Figures $8 \mathrm{c}$ and $8 \mathrm{~d}$ ).

\section{Comparison With Natural Granular Flows}

In this section, we compare the results of our laboratory experiments to what has been observed for natural granular flows and interpret the differences.

\subsection{Comparison Between Granular Flow Dynamics and Seismic Signal}

The spectrogram of the experimental granular flows has a cigar shape with an emergent onset, as observed for rockfalls, rock avalanches, and landslides in the field (e.g., Dammeier et al., 2011; Hibert et al., 2011; Hibert, Mangeney, et al., 2014; Hibert, Mangeney, et al., 2017; Moretti et al., 2012; Schneider et al., 2010) (Figures $2 \mathrm{c}$ and $2 \mathrm{~g}$ ). It is much more elongated for high slope angles $\theta$. The fact that the decay phase of the seismic amplitude lasts longer than the rise phase may thus be an indication that the landslide is propagating on a steep slope in the field. However, this observation may only apply to simple slope configurations with an approximately constant slope angle because the shape of the seismic signal generated by granular flows 
also depends on bed topography (Favreau et al., 2010; Lévy et al., 2015). In addition, as opposed to our simple laboratory case, the slope angle in the field often varies greatly along the path of the landslide from steep slopes close to the summit to more gentle slope angles in the runout area, which could change the flow duration and runout distance compared to those that would be observed on a slope with a constant angle (e.g., Sulpizio et al., 2016, and references therein). Extrapolating the slope angle from the shape of the recorded seismic signal may therefore be difficult in the field.

Recently, Hibert, Ekström, et al. (2017) investigated the high-frequency $(3-10 \mathrm{~Hz})$ seismic signals emitted by 12 large landslides of masses from $2 \times 10^{9} \mathrm{~kg}$ to $1.3 \times 10^{11} \mathrm{~kg}$ in various geological contexts. They reported a quantitative correlation between the maximum amplitude of the seismic envelope $E n v(t)$ and the maximum flow momentum $\left|M V_{X}\right|$, where $M$ is the total mass of the flow and $V_{X}$ the bulk speed of the center of mass in the $X$ direction. In contrast, we observed here that the maximum amplitude of the seismic envelope $\operatorname{Env}(t)$ does not correspond to the maximum of the speed of the granular flow in the $X$ direction, but rather to the maximum of speed in the $Z$ direction (Figures 7a to 7c). The rise phase in the laboratory seismic signals may be different from what is observed in the field because, during flow initiation in the field, the cohesive granular mass is fractured into smaller particles. We find another indication that the rise phase is different for experimental and natural granular flows when we compare their durations in terms of the characteristic flow duration $\tau_{c}=\sqrt{h_{0} /(g \cos \theta}$ ) (Mangeney et al., 2010). In the laboratory, with $h_{0} \simeq 2 \mathrm{~cm}$ and $\theta=0^{\circ}-20^{\circ}$, $\tau_{c} \simeq 0.05 \mathrm{~s}$, and the rise phase lasts approximately $3 \tau_{c}$, which is in agreement with previous larger-scale granular flow experiments (e.g., Farin et al., 2014; Mangeney et al., 2010). On the other hand, in the field, examples of values in the crater of Piton de la Fournaise volcano (Hibert et al., 2011) are $h_{0}=10 \mathrm{~m}$ and $\theta=35^{\circ}$, thus $\tau_{c} \simeq 1 \mathrm{~s}$ and the rise phase lasts $\sim 10 \tau_{c}>3 \tau_{c}$. Therefore, for future studies, it may be more relevant to compare seismic signal generation by granular flow between laboratory, model, and field observations during the propagation of the flow, rather than during the initiation and stopping phases. More work is also needed to understand how the change of slope angle along the runout path of the granular flow affects the generated seismic signal (Favreau et al., 2010; Lévy et al., 2015).

\subsection{Characteristic Frequencies of the Signals Generated by Impacts and Granular Flows}

The high-frequency $(>1 \mathrm{~Hz}$ ) seismic signal generated by granular flows is thought to be due to the multiple impacts of the particles on the bed. We recorded the signal generated by the impact of a single steel bead of diameter $2 \mathrm{~mm}$ on the rough PMMA plate (Figure 9a). The generated frequencies range between $1 \mathrm{kHz}$ and $18 \mathrm{kHz}$, with a mean frequency $f_{\text {mean }} \approx 5.6 \mathrm{kHz}$ (Figure 9b), which is similar to that of the signal generated by the granular flows (Figure 8c). Huang et al. (2007) also observed that the seismic signal generated by a debris flow has frequencies (from $10 \mathrm{~Hz}$ to $100 \mathrm{~Hz}$ ) similar to that of the signal generated by the impact of a single particle (from $10 \mathrm{~Hz}$ to $150 \mathrm{~Hz}$ ) at the same distance from the seismic station. In addition, Helmstetter and Garambois (2010) measured signal frequencies in the same range, from $1 \mathrm{~Hz}$ up to $100 \mathrm{~Hz}$, and with an energy peak of around $10-20 \mathrm{~Hz}$, for impacts of a single rock and for rockfalls of several hundreds of cubic meters involving many rocks. As stated earlier, if the impacts of particles are uncorrelated, the shape of the amplitude spectrum should not change from that of a single impact (Tsai et al., 2012). In this case, the frequency of the signal generated by a granular flow should be similar to that generated by a single impact. Lower frequencies may be observed in dense granular flows because of the presence of longer particle contacts that during collisions, for example, chains of forces (e.g., Estep \& Dufek, 2012), could increase the duration of the particle impacts compared to the case of a single particle bouncing down the slope. We therefore deduce that the frequencies of the signal emitted by an impact provide an upper bound for the frequencies generated by a granular flow composed of particles of the same size.

In our impact and granular flow experiments, we record higher signal frequencies, between $1 \mathrm{kHz}$ and $20 \mathrm{kHz}$, than those recorded in the field for block impacts and natural rockfalls, landslides, and debris flows, which are typically between $1 \mathrm{~Hz}$ and $150 \mathrm{~Hz}$ (e.g., Dammeier et al., 2011; Deparis et al., 2008; Farin et al., 2015; Helmstetter \& Garambois, 2010; Hibert et al., 2011; Hibert, Malet, et al., 2017; Huang et al., 2007; Vilajosana et al., 2008). This difference can be explained by the fact that the typical duration $T_{c}$ of particle impacts (equation (15)) is shorter in the laboratory than in the field. Indeed, the characteristic frequency $f_{c}$ of the generated seismic signal is inversely proportional to the duration $T_{c}$ of the impact (Farin et al., 2015). In the laboratory experiments, the elastic parameters of PMMA and steel materials lead to a characteristic frequency $f_{c} \sim 1 / T_{c} \simeq 15 \mathrm{kHz}$ for particles of typical radius $R \sim 1 \mathrm{~mm}$ (Table 2). This theoretical estimate is close to the frequency $f_{\text {mean }} \simeq 13 \mathrm{kHz}$ we measure in our experiments for an impact on the smooth surface of the plate (blue dashed line in Figure 9b). In contrast, in the field, the soil is loose, rock materials are brittle and the typical 

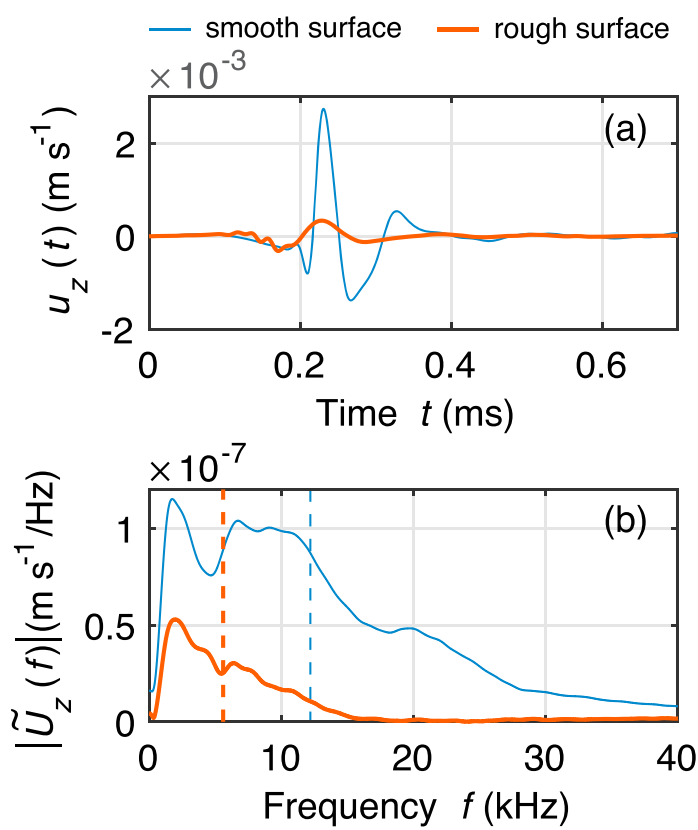

Figure 9. (a) Seismic signal $u_{Z}(t)$ generated at $r=10 \mathrm{~cm}$ from an impact of a 2-mm steel bead dropped from height $h=15 \mathrm{~cm}$ on the smooth PMMA plate (thin blue line) and on the same plate after the surface was made rough by gluing a layer of particles on its surface (thick red line). (b) Amplitude spectrum $\left|\tilde{U}_{Z}(f)\right|$ corresponding to the seismic signal recorded on the smooth and rough plates. The dashed vertical lines represent the mean frequency $f_{\text {mean }}$ of the amplitude spectrum. PMMA = poly(methyl methacrylate).

elastic modulus is $E^{*} \simeq 10^{7} \mathrm{~Pa}$ (Geotechdata.info, 2013). Impactor diameters can also be of the order of millimeters in the field, but impacts of these particles do not radiate sufficient seismic energy to be detected by the seismic stations. Only impacts of larger blocks $(0.1-1 \mathrm{~m})$ are detected and, combined with the lower values of $E^{*}$, we estimate that these impacts generate low characteristic frequencies $f_{c} \sim 1 / T_{c}$ from $1 \mathrm{~Hz}$ to $130 \mathrm{~Hz}$ (for typical rock parameters, see Table 2), in agreement with the frequencies observed in the field.

Hertz's duration of impact $T_{c}$ is therefore a good characteristic time to use to upscale the frequency $f_{c} \sim 1 / T_{c}$ of seismic signals generated by granular flows measured in the laboratory to those measured in the field. This approach, however, can be imprecise because the frequencies of the seismic signal emitted by an impact also strongly depend on the roughness of the ground, which is not taken into account in Hertz's expression of the impact time $T_{c}$ (equation (15)). For example, when we drop a steel bead on the smooth PMMA plate (without the layer of steel beads glued on its surface), the mean frequency of the emitted signal is more than 2 times higher $\left(f_{\text {mean }} \simeq 13 \mathrm{kHz}\right.$, blue line in Figure $9 \mathrm{~b}$ ) than when the particle impacts the rough bed

Table 2

Comparison of Characteristic Time and Frequency in the Laboratory and in the Field ${ }^{\mathrm{a}}$

\begin{tabular}{lcc}
\hline Parameter & Laboratory & Field \\
\hline$E^{*}(\mathrm{~Pa})$ & $10^{9}$ & $10^{7}$ \\
$\rho_{s}\left(\mathrm{~m}^{3} / \mathrm{kg}^{3}\right)$ & 7,800 & $2,000-3,000$ \\
$R(\mathrm{~m})$ & $10^{-3}$ & $0.1-1$ \\
$V_{Z}\left(\mathrm{~m} / \mathrm{s}^{1}\right)$ & 1 & $1-10$ \\
$T_{C}(\mathrm{~s})$ & $6.7 \times 10^{-5}$ & $8 \times 10^{-3}$ to 1 \\
$f_{c}(\mathrm{~Hz})$ & 15,000 & $1-130$ \\
\hline
\end{tabular}

${ }^{a}$ Comparison of the theoretical characteristic frequency $f_{c}=1 / T_{c}$, where $T_{c}$ is the Hertz duration of the impact (equation (15)), of the signal generated by an impact in the laboratory and in the field. $E^{*}$ is the equivalent Young's modulus and $\rho_{s}, R$, and $V_{Z}$ are the impactor density, radius, and speed. Parameters from the field are from Iverson (1997). 
$\left(f_{\text {mean }} \simeq 5.6 \mathrm{kHz}\right.$, red line in Figure $\left.9 \mathrm{~b}\right)$. In the field, the measured mean frequency $f_{\text {mean }}$ should also decrease as the distance between the seismic source and the seismic station increases because high-frequency energy attenuates more rapidly than low-frequency energy in heterogeneous media (Aki \& Richards, 1980).

\subsection{Seismic Efficiency and Scaling Laws of Granular Flows}

We now compare the seismic efficiency (ratio of radiated seismic energy $W_{\mathrm{el}}$ to potential energy lost $\Delta E_{p}$ ) of a single impact with that of a granular flow in the laboratory. We determined the seismic energy $W_{\text {el }}$ radiated during the impact of a single 2-mm steel bead dropped from height $h=21 \mathrm{~cm}$ on the same rough PMMA plate as in the granular flow experiments from the integral of the energy flux recorded at the sensor, similar to what is done in the field (Farin et al., 2015; Hibert et al., 2011; Vilajosana et al., 2008) and in the impact experiments of Farin et al. (2015)

$$
W_{\mathrm{el}}=\int_{0}^{+\infty} 2 r h_{p} \rho_{p} v_{g}(\omega)\left|\tilde{U}_{Z}(r, \omega)\right|^{2} \exp (\gamma(\omega) r) \mathrm{d} \omega,
$$

where $\tilde{U}_{Z}(r, \omega)$ is the Fourier transform of the first wave arrival $u_{z}(t)$, recorded by the sensors before the return of the reflections off the plate side boundaries (shown in Figure 9 ), $r=10 \mathrm{~cm}$ is the distance between the sensor and the impact, $h_{p}=1 \mathrm{~cm}$ is the plate thickness, $\rho_{p}=1,180 \mathrm{~kg} / \mathrm{m}^{3}$ is the plate density, $\omega=2 \pi f$ is the circular frequency, $v_{g}(\omega)$ is the group speed, and $\gamma(\omega)$ is the characteristic coefficient for anelastic attenuation of energy with distance (in units of $\mathrm{m}^{-1}$ ). Farin et al. (2016) determined, for a PMMA plate, $v_{g}(\omega) \simeq 11.7 \sqrt{f} \mathrm{~m} / \mathrm{s}$ and $\gamma(\omega) \simeq 1 \mathrm{~m}^{-1}$ for $k h<1$ and $v_{g}(\omega) \simeq 1,400 \mathrm{~m} / \mathrm{s}$ and $\gamma(\omega)=4.8 \times 10^{-3} f^{2 / 3} \mathrm{~m}^{-1}$ for $k h>1$, where $k$ is the wave number. Note that the calculation of the radiated seismic energy $W_{\mathrm{el}}$ in equation (16) is carried out in the frequency domain, while calculations are usually made in time domain in the field (Hibert et al., 2011; Vilajosana et al., 2008); however, this is equivalent due to Parseval's Theorem. We obtain $W_{\mathrm{el}} \simeq 2.7 \times 10^{-6} \mathrm{~J}$ for the seismic energy radiated by a single-particle impact on the rough PMMA plate. The potential energy lost during the impact is $\Delta E_{p}=\left(1-e^{2}\right) m g h \simeq 9 \times 10^{-5} \mathrm{~J}$, with $m$, the mass of the particle and $e \simeq 0.4$, the coefficient of restitution. Thus, for one particle impact on the rough plate, the seismic efficiency is $W_{\mathrm{el}} / \Delta E_{p} \simeq 0.03$. At the same slope angle $\theta=0^{\circ}$, the seismic efficiency is $W_{\mathrm{el}} / \Delta E_{p} \simeq 3.3 \times 10^{-4}$ for a granular flow composed of the same particles (Figure 6e). A granular flow is then about 90 times less seismically efficient than a single impact.

In order to interpret the difference in transmission of seismic energy for the impact of a single particle versus a granular flow, we detail the energy budget during an impact. The proportion of the initial potential energy of the particle, $E_{p}$, which is not returned to the particle for its rebound or rotation modes $\left(E_{p}^{\prime}\right)$ is either radiated in the form of seismic waves in the plate $\left(W_{\mathrm{el}}\right)$, dissipated by a viscoelastic process (heat loss) in the vicinity of the impact $\left(W_{\text {visc }}\right.$; Falcon et al., 1998) or lost in irreversible fracturing of the impactor or of the bed surface $\left(W_{\text {plast }} ;\right.$ Davies, 1949). The potential energy lost by the particle at impact is then:

$$
\Delta E_{p}=E_{p}-E_{p}^{\prime}=W_{\mathrm{el}}+W_{\text {visc }}+W_{\text {plast }}
$$

The only energy that we can measure in the present experiments is the radiated seismic energy $W_{\mathrm{el}}$, which depends, in part, on other sources of loss. For example, for a given loss of potential energy $\Delta E_{p}$, the more energy that is spent in irreversible deformation on a rough surface $\left(W_{\text {plast }}\right)$, the less that is available for transmission as elastic waves $\left(W_{\mathrm{el}}\right)$. Because of particle collisions and friction in granular flows, a larger proportion of the potential energy loss $\Delta E_{p}$ is expected to be dissipated within the flow by inelastic processes, resulting in a smaller amount of radiated seismic energy $\left(W_{\mathrm{el}}\right)$ compared to the impact of a single particle dropped on the bed. Our measurements of seismic efficiency $\left(W_{\mathrm{el}} / \Delta E_{p}\right)$ on the rough PMMA plate at a $0^{\circ}$ slope indicate that inelastic processes account for about $99.967 \%$ of the potential energy that is lost during a granular flow, compared to $97 \%$ for the impact of a single grain, as reported above. Note that these values should change if different plate and impactor materials or different particle diameters are used because these parameters critically affect the seismic efficiency for particle impacts (Farin et al., 2016).

Only a few measurements of the seismic efficiency $W_{\mathrm{el}} / \Delta E_{p}$ of rock impacts and granular flows exist in the literature and no study has compared the seismic efficiency for single particle impacts and granular flows at the same site. From site to site, the reported ratios $W_{\mathrm{el}} / \Delta E_{p}$ vary over several orders of magnitude from $10^{-6}$ to 0.25 (Deparis et al., 2008; Farin et al., 2015; Hibert et al., 2011; Hibert, Malet, et al., 2017; Lévy et al., 2015; Vilajosana et al., 2008). In the present laboratory experiments, we observed that the seismic efficiency $W_{\text {el }} / \Delta E_{p}$ of a granular flow decays by a factor of 2 , from $3.3 \times 10^{-4}$ to $1.7 \times 10^{-4}$, when slope angle $\theta$ is increased 
(a)

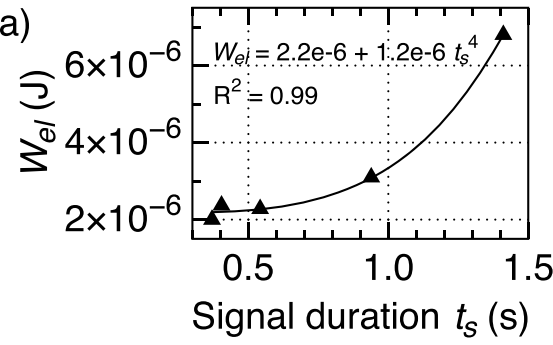

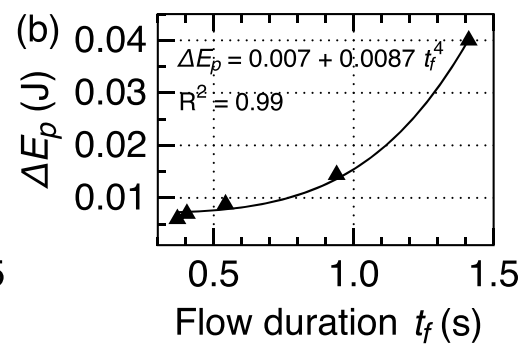

Figure 10. (a) Radiated seismic energy $W_{\mathrm{el}}$ as a function of the signal duration $t_{s}$. (b) Potential energy lost $\Delta E_{p}$ as a function of the flow duration $t_{f}$.

from $0^{\circ}$ to $20^{\circ}$ (Figure 6e). This factor of 2 can only explain a very small part of the range of seismic efficiencies observed in the field. Another cause of uncertainty in the ratio $W_{\mathrm{el}} / \Delta E_{p}$ could come from the uncertainty in the elastic parameters of the ground, for example, the density $\rho_{p}$ and the wave speed $v_{g}$ that are present in the calculation of $W_{\text {el }}$ (equation (16)). However, the major cause of the discrepancy of the seismic efficiency $W_{\mathrm{el}} / \Delta E_{p}$ in the field is likely the roughness of the contact between the impactor and the ground. For example, for an impact on the smooth surface of the PMMA plate, we determine a seismic efficiency $W_{\mathrm{el}} / \Delta E_{p} \simeq 0.26$ (using equation (16) with the signal shown in Figure 9), which is about 10 times larger than that measured when the plate is covered with a layer of beads glued on its surface $\left(W_{\mathrm{el}} / \Delta E_{p} \simeq 0.03\right)$. The measured seismic power is 10 times lower when the particle impacts the rough bed probably because some of the energy radiated at impact propagates in the glued particles and is not transmitted to the plate or because more energy is lost in deformation on the rough bed [ $W_{\text {plast }}$ in the budget equation (17)]. Similarly, Farin et al. (2015) noted that the seismic efficiency $W_{\mathrm{el}} / \Delta E_{p}$ measured for single-boulder impacts (from $300 \mathrm{~kg}$ to $4,800 \mathrm{~kg}$ ) on a hard basalt slope in Tahiti, French Polynesia, was on the average 1 order of magnitude larger $\left(10^{-2}\right)$ for impacts of rock blocks on bedrock than for impacts on loose sediment $\left(10^{-3}\right)$. The seismic efficiencies measured for these boulder impacts $\left(W_{\mathrm{el}} / \Delta E_{p} \simeq 10^{-4}-10^{-1}\right)$ are several orders of magnitude larger than that estimated by Hibert, Malet, et al. (2017) for impacts of boulders of similar sizes, ranging from $76 \mathrm{~kg}$ to $472 \mathrm{~kg}$, on soft black marls in the French Alps $\left(W_{\mathrm{el}} / \Delta E_{p} \simeq 10^{-6}\right)$. Kean et al. (2015) observed that the seismic power radiated by a debris flow propagating on a thin layer of sediment was about 2 orders of magnitude lower than for a debris flow of similar characteristics propagating directly on bedrock. Similarly, Pérez-Guillén et al. (2016) also measured a decrease by 2 orders of magnitude of the seismic energy radiated by snow avalanches when the thickness of the snow cover above the bed increased from $0 \mathrm{~m}$ to $4 \mathrm{~m}$. These field observations are in good agreement with what was found at the laboratory scale by Bachelet et al. (2018), who reported a decrease of 2 orders of magnitude of the seismic efficiency for a particle impact on a rough surface compared to the same impact on an erodible bed with a thickness of 10 particle diameters. More generally, they found that the seismic energy radiated by a particle impact decreased exponentially as the thickness of the erodible bed increased. In conclusion, the several orders of magnitude variation in seismic efficiency that have been observed in the field may predominately reflect the state of the bed on which the granular flow propagates (smooth, rough, or erodible). In our experiments, seismic efficiency is much less affected by variation of the slope angle (causing only a factor of 2 change in efficiency over the range of slopes examined). That said, the effect of particle diameter, flow mass, and initial aspect ratio on the seismic efficiency $W_{\mathrm{el}} / \Delta E_{p}$ warrants further investigation.

Interestingly, we observe that the seismic energy $W_{\text {el }}$ radiated by granular flows increases with the fourth power of the duration $t_{s}$ of the seismic signal (i.e., $t_{s}^{4}$ ) and the potential energy lost $\Delta E_{p}$ during the granular collapse scales with the fourth power of the duration $t_{f}$ of the flow (i.e., $t_{f}^{4}$; Figures $10 \mathrm{a}$ and $10 \mathrm{~b}$ ). The seismic signal generated by the granular flow is comprised between the seismic signals generated by the first and the last particle impacts in the flow. Before and after these impacts, we can reasonably assume that the particles are not moving, with an accuracy of a few milliseconds. In addition, the duration of the coda of the signal generated by the last impact on the PMMA plate is at most a few milliseconds (see Figure $5 c$ of ; Farin et al., 2015), which is negligible compared to the duration of the granular flows, from several tenths of seconds to several seconds (Figures $2 \mathrm{~b}$ and $2 \mathrm{f}$ ). Consequently, we can consider that the duration of the recorded seismic signal $t_{s}$ is equal to the duration $t_{f}$ of the granular flow with an accuracy of a few milliseconds ( $<1 \%$ of the flow duration). It is also generally accepted that $t_{s} \simeq t_{f}$ for granular flows in the field if the seismic signal is recorded close to the event because the duration of the coda due to scattering in the ground is negligible compared 
to the flow duration (Dammeier et al., 2011; Deparis et al., 2008; Hibert et al., 2011; Vilajosana et al., 2008; see also Figure 2 of Hibert, Mangeney, et al., 2017). Thus, both the increase of the radiated seismic energy $W_{\mathrm{el}}$ and the potential energy lost $\Delta E_{p}$ when the slope angle $\theta$ is increased are due to an increase in the flow duration $t_{f}$. This scaling law with the fourth power of flow duration $t_{f}$ was predicted analytically by Hibert (2012) for a granular collapse on a flat inclined plane. In the field, because the slope angle decreases during flow propagation, the radiated seismic energy $W_{\mathrm{el}}$ is observed to vary with signal duration $t_{s}$ to the power of between 1.3 and 2.7 (Hibert et al., 2011; Lévy et al., 2015).

\section{Conclusions}

We have conducted simple granular column collapse experiments on a flat PMMA plane inclined at various angles with simultaneous measurements of the flow dynamics and of the generated seismic signal. We made some strong assumptions (constant flow width and density and uncorrelated impacts) to be able to determine the potential energy lost and the radiated seismic energy during the granular flows. Nevertheless, we expect the uncertainties on the determined energies to be less than an order of magnitude. We will now address the questions posed in section 1.

1. Seismic efficiency (the proportion of lost potential energy that is converted into radiated seismic energy) exhibited a twofold decrease (from $0.033 \%$ to $0.017 \%$ ) as slope angle increased from $0^{\circ}$ to $20^{\circ}$ in our experiments. The dependence on the slope angle is probably due to the fact that particle impacts are directed more normally to the slope, and thus more seismically efficient, at small slope angles. Although seismic efficiency did not vary in time during the granular flow, we note that efficiency is expected to vary with differences in particle diameter and material composition of both the particles and the PMMA plate, as demonstrated by single-grain impacts (Farin et al., 2016).

2. The decrease in seismic efficiency with increased slope may partially explain the scatter in seismic efficiencies observed in field studies of landslides $\left(10^{-6}\right.$ to 0.25$)$ because field slopes may not be well constrained and can vary along runout paths. However, we also observed that the seismic efficiency is about 10 times lower for a particle impact on a rough surface than on a smooth surface and deduced that the seismic efficiency of granular flows mostly depends on the state of the bed on which the flow propagates (smooth, rough, or erodible). The signal frequencies generated by the experimental granular flows are 100 to 1,000 times higher than those recorded for natural events. We theoretically accounted for this difference by computing, for the laboratory experiments and the field, a characteristic signal frequency defined as the inverse of the duration of impact predicted by Hertz's model (Hertz, 1882). We concluded that Hertz's impact duration is an appropriate characteristic time for upscaling the frequencies of seismic signals recorded in the laboratory to those observed in the field.

3. In our experiments, the seismic efficiency of granular flows was about 90 times lower than for the impact of a single particle for material of the same diameter, suggesting that granular flows lose more potential energy in inelastic processes, such as viscous dissipation and irreversible deformation of the particles or the ground (with inelastic processes accounting for $\sim 99.967 \%$ of the lost potential energy in our granular flow experiments compared to $\sim 97 \%$ for the impact of a single particle). The frequencies of the signal emitted by our granular flows were below $20 \mathrm{kHz}$, with a mean around $5 \mathrm{kHz}$, which is similar to that measured for the impact of a single particle of the same kind as the ones used in the granular flows. To estimate the frequency range that would be generated by a granular flow at a given site, one could drop rock blocks on the expected path of the landslide and determine the frequency content of the generated impulse signals. The sampling frequency used to monitor the subsequent granular flows should then be at least 2 times the maximum frequency emitted by the impacts of the rock blocks, taking into account the Nyquist-Shannon sampling theorem.

4. We do not observe any correlation in time and amplitude between the maximum of the seismic envelope generated by the granular flows and the maximum speed of the flow center of mass (nor of the flow front) in the downslope direction, in contrast with what was reported recently by Hibert, Ekström, et al. (2017) for large natural landslides. The reason for this difference may be that the initiation and stopping phases of the granular flows in the experiments are different than those for granular flows in the field. In the experiments, the granular mass is released suddenly when the reservoir containing the particles is removed and propagates on a slope of constant inclination until friction overcomes gravity. In contrast, in the field, a granular flow is initiated when a consistent mass is destabilized and collapses into pieces, often stopping when the 
slope angle decreases. On the other hand, in our experiments, the maximum value of the amplitude envelope coincides with the maximum speed of the center of mass in the direction normal to the slope, which may correspond with the maximum of the rate and speed of normal particle impacts. We conclude that future comparisons of experiments and field observations of seismic signals generated by granular flows should focus on the propagation of the granular flow rather than on the initiation and stopping phases.

5. We observed that the shape of the temporal variation of the signal envelope, signal frequencies, and cumulative radiated seismic energy changes above a critical slope angle between $10^{\circ}$ and $15^{\circ}$ (i.e., around half the friction angle of the granular material, which is about $27^{\circ}$ ). Indeed, the duration of the decay phase of the signal amplitude and frequencies does not vary much below the critical slope angle, but increases significantly for slope angles above the critical value. This change seems to correspond to a change of dynamics for the granular flow from a dense to a more dilute agitated flow at the end of the deceleration phase. The duration of this agitated regime increases as the slope angle is increased. This suggests that it may be possible to detect, in the generated seismic signal, a change in the dynamics of granular flows when the slope angle is increased.

Our experiments demonstrate that the seismic monitoring of granular flows is a relevant tool for obtaining insights regarding flow dynamics. Our experimental data can be useful to test future analytical and numerical models predicting the seismic signal generated by granular flows at different slope angles, both for field and industrial applications. More experiments are needed to investigate the role of the particle diameter, initial volume and aspect ratio of the granular column and the presence of an erodible bed on the frequency of the generated seismic signal and the seismic efficiency of granular flows. In particular, to make it possible to determine the potential energy of granular flows from the generated seismic signal, future work will be required to understand how a bed of loose sediment attenuates the transmission of seismic waves to the ground, depending on its nature and thickness.

\section{Appendix A: Seismic Energy Radiated During a Granular Flow}

In this Appendix, we derive the expression of the seismic energy $W_{\mathrm{el}}$ radiated during a granular column collapse from the seismic energy radiated during an individual impact in a diffuse field.

We assume that the individual impacts occurring during the granular collapse are uncorrelated and that the seismic energy $W_{\mathrm{el}}$ radiated by the granular flow is the sum of the energies $W_{i}$ radiated by the impacts $i$. Farin et al. (2016) showed that, for an individual impact,

$$
W_{i} \approx \rho_{p} v_{p} \overline{u_{z i}^{2}}
$$

where $\rho_{p}$ is the plate density and $V_{p}$ is its volume; $\overline{u_{z i}{ }^{2}}$ is the amplitude of the normal squared vibration speed at the instant of the impact $i$.

The seismic energy radiated by the granular flow is then

$$
W_{\mathrm{el}} \approx \rho_{p} v_{p} \sum_{i} \overline{u_{z i}{ }^{2}}
$$

It is not possible to determine the term $\overline{u_{z i}{ }^{2}}$ for each impact $i$ in the seismic signal emitted by a granular collapse. Therefore, we express the sum of the $\overline{u_{z i}{ }^{2}}$ as a function of the vibration speed $u_{z}(t)$ emitted by the granular flow (e.g., as shown in Figures $2 b$ and $2 f$ ).

The vibration speed $u_{z}(t)$ generated by the granular column collapse is the sum of the vibrations $u_{i}\left(t-t_{i}\right)$ emitted by each impact $i$ at time $t_{i}$

$$
u_{z}(t)=\sum_{i} u_{i}\left(t-t_{i}\right)
$$

The integral of its squared vibration is then given by

$$
\begin{aligned}
\int_{0}^{+\infty} u_{z}^{2}(t) \mathrm{d} t & =\sum_{i, j} \int_{0}^{+\infty} u_{i}\left(t-t_{i}\right) u_{j}\left(t-t_{j}\right) \mathrm{d} t \\
& =\sum_{i} \int_{0}^{+\infty} u_{i}^{2}\left(t-t_{i}\right) \mathrm{d} t+\sum_{i \neq j} \int_{0}^{+\infty} u_{i}\left(t-t_{i}\right) u_{j}\left(t-t_{j}\right) \mathrm{d} t .
\end{aligned}
$$


It can be shown that the amplitude of the first term in equation (A5) varies according to $\tau \Delta f$, while that of the second term, in the case of uncorrelated impacts, varies as a function of $\sqrt{\tau \Delta f}$, where $\tau$ is the characteristic time of energy dissipation and $\Delta f$ is the bandwidth of the emitted signal (Goodman, 2000). The amplitude of the ratio of the second term to the first term then varies according to $1 / \sqrt{\tau \Delta f}$. For a granular column collapse constituted of steel beads on a PMMA plate, $1 / \sqrt{\tau \Delta f} \approx 0.35$. Using a simple computation, we can estimate the number of uncorrelated impacts that occur during the time $\tau$. In the experiments, the column initial radius is $r_{0}=15 \mathrm{~mm}$ and the bead radius is $R=1 \mathrm{~mm}$. The approximate number of beads in a section parallel to the slope is then $r_{0}^{2} / R^{2} \approx 230$ beads. Assuming that the column collapses with the mean normal speed $V_{Z} \simeq 200 \mathrm{~mm} / \mathrm{s}$ without spreading itself, there are, in $1 \mathrm{~s}, V_{Z} / 2 R \simeq 200 / 2=100$ layers of beads colliding with the surface (i.e., $100 \times 230=23,000$ bead impacts). For PMMA, $\tau \approx 1.2 \times 10^{-3} \mathrm{~s}$. Therefore, during time $\tau$, there are about $23,000 \times 1.2 \times 10^{-3} \approx 27$ uncorrelated impacts. We can then neglect the term of uncorrelated impacts (second term) in equation (A5).

Consequently,

$$
\int_{0}^{+\infty} u_{z}^{2}(t) \mathrm{d} t \approx \sum_{i} \int_{0}^{+\infty} u_{i}^{2}\left(t-t_{i}\right) \mathrm{d} t
$$

For an individual impact, Farin et al. (2016) showed that when the wave field is diffuse, the averaged squared vibration speed decays exponentially with time as

$$
u_{i}^{2}\left(t-t_{i}\right)=\overline{u_{z i}^{2}} \exp \left(-\frac{t-t_{i}}{\tau}\right),
$$

where $\overline{u_{z i}{ }^{2}}$ is the amplitude of the squared vibration speed at instant $t_{i}$ of impact $i$. Integrating $u_{i}^{2}\left(t-t_{i}\right)$ in equation (A6), we thus obtain

$$
\int_{0}^{+\infty} u_{z}^{2}(t) \mathrm{d} t \approx \tau \sum_{i} \overline{u_{z i}^{2}}
$$

To conclude, from equations (A2) and (A8), the seismic energy $W_{\mathrm{el}}$ radiated during a granular column collapse on a thin plate is given by

$$
W_{\mathrm{el}} \approx \frac{\rho_{p} V_{p}}{\tau} \int_{0}^{+\infty} u_{z}^{2}(t) \mathrm{d} t
$$

and the cumulative radiated seismic energy is, at time $t$,

$$
W_{\mathrm{el}}(t) \approx \frac{\rho_{p} V_{p}}{\tau} \int_{0}^{t} u_{z}^{2}\left(t^{\prime}\right) \mathrm{d} t^{\prime}
$$

Dividing by time $\tau$ in equation (A10) allows us to correct our estimate of the radiated seismic energy $W_{\mathrm{el}}$ from the additional energy coming from the wave reflections off the lateral sides of the plate and thus to obtain a good estimate of $W_{\mathrm{el}}$. If the characteristic time $\tau$ of energy dissipation in the plate is longer, the emitted waves propagate along a longer distance before being dissipated. Consequently, more side reflections are recorded by the sensors and the corrective factor $1 / \tau$ is higher.

\section{Appendix B: Comparison of Radiated Seismic Power With Lost Power}

In this Appendix, we compare the radiated seismic power $\Pi_{\mathrm{el}}(t)$, which is the time derivative of the cumulative radiated seismic energy $W_{\mathrm{el}}(t)$ (Figure $\mathrm{B} 1 \mathrm{a}$ ), with the time derivatives of the potential energy lost $\Delta E_{p}(t)$ (Figure $\mathrm{B} 1 \mathrm{~b}$ ) and of the total energy lost $E_{\text {tot }}(t)=\Delta E_{p}(t)+E_{c}(t)$ (Figure B1c). The maximum of the radiated power $\Pi_{\mathrm{el}}(t)$ matches in time the maximum of the energy derivative $\mathrm{d} \Delta E_{p}(t) / \mathrm{d} t$ and $\mathrm{d} \Delta E_{\mathrm{tot}}(t) / \mathrm{d} t$, at around $t \simeq 0.15 \mathrm{~s}$. The normalized cumulative curves also match well in time and the shape of the cumulative curves change after a critical slope angle $\theta$ between $10^{\circ}$ and $15^{\circ}$, similar to what is observed for the cumulated energies (Figures $6 \mathrm{~d}$ and B1d). Moreover, the cumulative radiated seismic power $\Pi_{\mathrm{el}}(t)$ matches better with the total lost power $\mathrm{d} \Delta E_{\text {tot }}(t) / \mathrm{d} t$ than with the derivative $\mathrm{d} \Delta E_{p}(t) / \mathrm{d} t$ of the potential energy lost. 


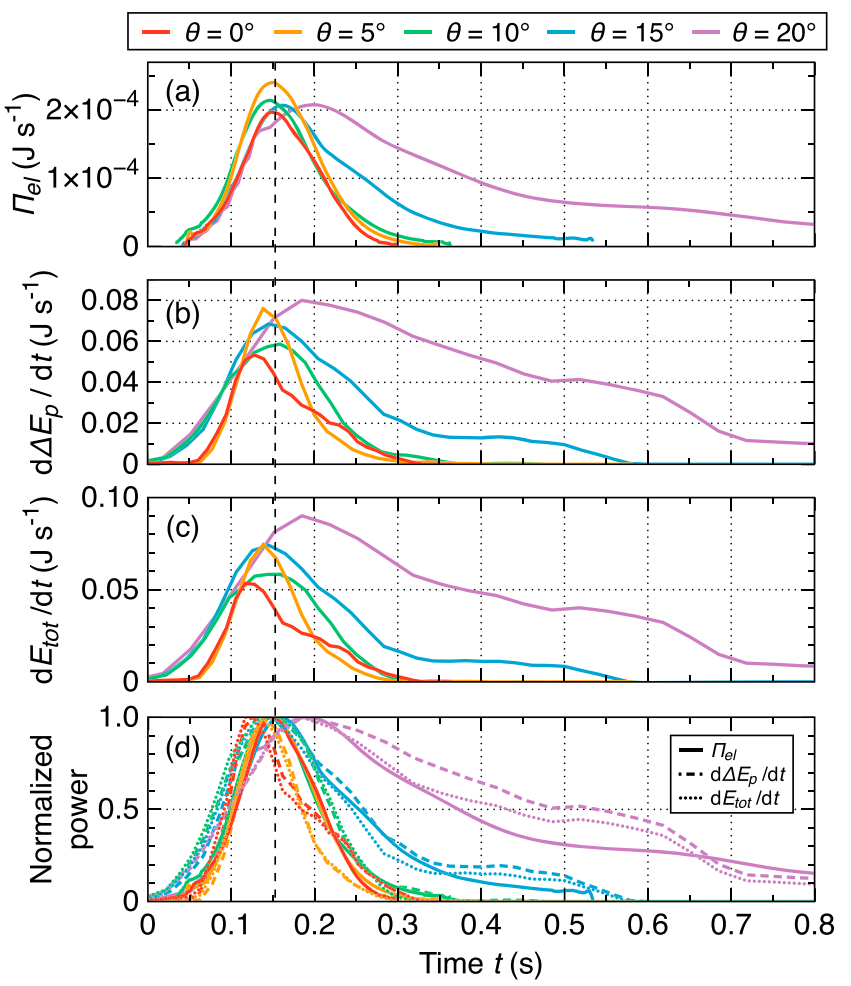

Figure B1. (a) Radiated seismic power $\Pi_{\mathrm{el}}(t)$ [derivative of the radiated seismic energy $W_{\mathrm{el}}(t)$ ]. (b) Time derivative of the potential energy lost $\Delta E_{p}(t)$. (c) Time derivative of the total energy lost $E_{\mathrm{tot}}(t)=\Delta E_{p}(t)+E_{c}(t)$. (d) Normalized cumulative power as a function of time $t$ for different slope angles (different colors).

\section{Notation}

a Aspect ratio $a=h_{0} / D_{0}(-)$.

$D_{0}, h_{0}$ Initial diameter and thickness of the granular column (m).

$d, R$ Bead diameter and radius $(\mathrm{m})$.

$E_{p}, E_{p}^{\prime}$ Potential energy and potential energy of a particle after rebound (J).

$\Delta E_{p}, E_{c}, E_{\text {tot }}$ Potential energy lost, bulk kinetic energy, and total energy lost (J).

$\operatorname{Env}(t)$ Amplitude envelope of the seismic signal ( $\mathrm{m} / \mathrm{s})$.

$E_{i}, E_{g}, E^{*}$ Young modulii of the impactor and the ground and equivalent elastic modulus $(\mathrm{Pa})$.

$f, \omega$ Frequency and angular frequency $\left(s^{-1}\right)$.

$f_{\text {mean }}, f_{\text {max }}, f_{c}$ Mean and maximum frequencies of the signal and characteristic frequency $f_{c}=1 / T_{c}(\mathrm{~Hz})$.

$g$ Gravitational acceleration $\left(\mathrm{m} / \mathrm{s}^{2}\right)$

$h(X, t), I(Z, t)$ Thickness and length profiles of the flow contour in the $(X, Y=0, Z)$ plane $(\mathrm{m})$.

$h_{p}, V_{p}$ Thickness $(\mathrm{m})$ and volume $\left(\mathrm{m}^{3}\right)$ of the plate.

$k$ Wave number $\left(\mathrm{m}^{-1}\right)$.

$M$ Mass of the granular flow $(\mathrm{kg})$.

$m$ Mass of a particle $(\mathrm{kg})$.

$r$ Distance from the impact $(\mathrm{m})$.

$T_{c}$ Duration of an impact (s) (equation (15)).

$t$ Time (s).

$t_{s}, t_{f}$ Duration of the seismic signal and duration of the flow motion (s).

$u_{i}, a_{i}$ Vibration speed $(\mathrm{m} / \mathrm{s})$ and acceleration $\left(\mathrm{m} / \mathrm{s}^{2}\right)$ of the plate surface in the direction $i$.

$\tilde{U}_{i}$ Time Fourier transform of $u_{i}(\mathrm{~m} / \mathrm{s} / \mathrm{Hz})$.

$v_{i}^{\text {COM }}$ Speed of the center of mass in the $i$ direction $(\mathrm{m} / \mathrm{s})$.

$V_{\text {front }}, V_{\text {summit }}$ Speeds of the flow front and summit $(\mathrm{m} / \mathrm{s})$.

$V_{Z}$ Impact speed of individual particles in the $Z$ direction $(\mathrm{m} / \mathrm{s})$.

$v_{g}(\omega)$ Group speed in the plate $(\mathrm{m} / \mathrm{s})$. 


\section{Acknowledgments}

This work was supported by the Agence Nationale de la Recherche ANR LANDQUAKES, REALISE, ITN FLOWTRANS, and ERC Contract ERC-CG-2013-PE10-617472 SLIDEQUAKES. The data used are shown in the figures. We are grateful to our three anonymous reviewers for their valuable comments that greatly improved our initial manuscript.
$W$ Width of the granular flow in the $Y$ direction $(\mathrm{m})$.

$W_{\text {el }}$ Seismic energy radiated during the impact $(\mathrm{J})$.

$W_{\text {visc }}, W_{\text {plast }}$ Energy lost in viscous dissipation and in irreversible deformation $(\mathrm{J})$.

$X, Y, Z$ Coordinates in the reference frame of the inclined plate $(\mathrm{m})$.

$X^{\prime}, Y^{\prime}, Z^{\prime}$ Coordinates in the reference frame of the laboratory $(\mathrm{m})$.

$X^{\mathrm{COM}}, Z^{\mathrm{COM}}$ Coordinates of the flow center of mass in the $X$ and $Z$ directions $(\mathrm{m})$.

$X^{\text {front }}, Z^{\text {summit }}$ Coordinates of the flow front and flow maximum height in the $X$ and $Z$ directions $(\mathrm{m})$.

$\Delta f$ Bandwidth of the seismic signal $(\mathrm{Hz})$.

$\delta$ Friction angle (deg).

$\theta$ Slope angle (deg).

$v_{i}, v_{g}$ Poisson ratios of the impactor and the ground (-).

$\rho_{s}, \rho_{p}$ Densities of the beads and of the plate $\left(\mathrm{kg} / \mathrm{m}^{3}\right)$.

$\tau, \gamma$ Characteristic time (s) and coefficient $(1 / \mathrm{m})$ of energy attenuation.

$\tau_{c}$ Characteristic duration of a granular flow (s).

\section{References}

Aki, K., \& Richards, P. (1980). Quantitative seismology: Theory and methods (Vol. 1). San Francisco, CA: W. H. Freeman.

Allstadt, K. (2013). Extracting source characteristics and dynamics of the August 2010 Mount Meager landslide from broadband seismograms. Journal of Geophysical Research: Earth Surface, 118, 1472-1490. https://doi.org/10.1002/jgrf. 20110

Andreotti, B., Forterre, Y., \& Pouliquen, O. (2013). Granular media: Between fluid and solid (Vol. 1). Cambridge UK: Cambridge University Press.

Arattano, M. (2000). On debris flow front evolution along a torrent. Physics and Chemistry of the Earth, 25(9), 733-740. https://doi.org/10.1016/S1464-1909(00)00094-0

Bachelet, V., Mangeney, A., De Rosny, J., Toussaint, R., \& Farin, M. (2018). Elastic wave generated by granular impact on rough and erodible surfaces. Journal of Applied Physics, 123, 044901. https://doi.org/10.1063/1.5012979

Balmforth, N. J., \& Kerswell, R. R. (2005). Granular collapse in two dimensions. Journal of Fluid Mechanics, 538(1), 399-428. https://doi.org/10.1017/S0022112005005537

Brodsky, E., Gordeev, E., \& Kanamori, H. (2003). Landslide basal friction as measured by seismic waves. Geophysical Research Letters, 30(24), 2236. https://doi.org/10.1029/2003GL018485

Crampin, S. (1965). Higher modes of seismic surface waves: Second Rayleigh mode energy. Journal of Geophysical Research, 70(20), 5135-5143. https://doi.org/10.1029/JZ070i020p05135

Dammeier, F., Moore, J. R., Haslinger, F., \& Loew, S. (2011). Characterization of alpine rockslides using statistical analysis of seismic signals. Journal of Geophysical Research, 116, F04024. https://doi.org/10.1029/2011JF002037

Davies, R. M. (1949). The determination of static and dynamic yield stresses using a steel ball. Proceedings of the Royal Society of London 197(1050), 416-432. https://doi.org/10.1098/rspa.1949.0073

Delannay, R., Valance, A., Mangeney, A., Roche, O., \& Richard, P. (2017). Granular and particle-laden flows: from laboratory experiments to field observations. Journal of Physics D: Applied Physics, 50(5), 053001. https://doi.org/10.1088/1361-6463/50/5/053001

Deparis, J., Jongmans, D., Cotton, F., Baillet, L., Thouvenot, F., \& Hantz, D. (2008). Analysis of rock-fall and rock-fall avalanche seismograms in the French Alps. Bulletin of the Seismological Society of America, 98(4), 1781-1796. https://doi.org/10.1785/0120070082

Dufresne, A. (2012). Granular flow experiments on the interaction with stationary runout path materials and comparison to rock avalanche events. Earth Surface Processes and Landforms, 37(14), 1527-1541. https://doi.org/10.1002/esp.3296

Estep, J., \& Dufek, J. (2012). Substrate effects from force chain dynamics in dense granular flows. Journal of Geophysical Research, 117, F01028. https://doi.org/10.1029/2011JF002125

Falcon, E., Laroche, C., Fauve, S., \& Coste, C. (1998). Behavior of one inelastic ball bouncing repeatedly off the ground. European Physical Journal B, 3(1), 45-57. https://doi.org/10.1007/s100510050283

Farin, M. (2015). Études expérimentales de la dynamique et de l'émission sismique des instabilités gravitaires (PhD Thesis). Paris: IPGP.

Farin, M., Mangeney, A., de Rosny, J., Toussaint, R., Sainte-Marie, J., \& Shapiro, N. (2016). Experimental validation of theoretical methods to estimate the energy radiated by elastic waves during an impact. Journal of Sound and Vibration, 362, 176-202. https://doi.org/10.1016/j.jsv.2015.10.003

Farin, M., Mangeney, A., \& Roche, O. (2014). Fundamental changes of granular flow dynamics, deposition, and erosion processes at high slope angles: Insights from laboratory experiments. Journal of Geophysical Research: Earth Surface, 119, $504-532$. https://doi.org/10.1002/2013JF002750

Farin, M., Mangeney, A., Toussaint, R., de Rosny, J., Shapiro, N., Dewez, T., \& et al. (2015). Characterization of rockfalls from seismic signal: Insights from laboratory experiments. Journal of Geophysical Research: Earth Surface, 120, 7102-7137. https://doi.org/10.1002/2015JB012331

Favreau, P., Mangeney, A., Lucas, A., Crosta, G., \& Bouchut, F. (2010). Numerical modeling of landquakes. Geophysical Research Letters, 37, L15305. https://doi.org/10.1029/2010GL043512

GdR Midi (2004). On dense granular flows. The European Physical Journal E, 14(4), 341-365. https://doi.org/10.1140/epje/i2003-10153-0

Geotechdata.info (2013). Soil Young's modulus. Retrieved from http://www.geotechdata.info/parameter/soil-young\%27s-modulus.html, 2017-10-08

Goodman, J. (2000). Statistical optics(p. 572). Hoboken, NJ: Wiley Classics Library.

Helmstetter, A., \& Garambois, S. (2010). Seismic monitoring of Séchilienne rockslide (French Alps): Analysis of seismic signals and their correlation with rainfalls. Journal of Geophysical Research, 115, F03016. https://doi.org/10.1029/2009JF001532

Hertz, H. (1882). Über die Berührung fester elastischer Körper (On the vibration of solid elastic bodies). Journal für die reine und angewandte Mathematik, 92, 156-171. https://doi.org/10.1515/crll.1882.92.156

Hibert, C. (2012). Apport de l'écoute sismique pour l'étude des éboulements du cratère Dolomieu, Piton de la Fournaise (île de la Réunion) (PhD Thesis). Paris: IPGP.

Hibert, C., Ekström, G., \& Stark, C. (2014). Dynamics of the Bingham Canyon Mine landslides from seismic signal analysis. Geophysical Research Letters, 41, 4535-4541. https://doi.org/10.1002/2014GL060592 
Hibert, C., Ekström, G., \& Stark, C. P. (2017). The relationship between bulk-mass momentum and short-period seismic radiation in catastrophic landslides. Journal of Geophysical Research: Earth Surface, 122, 1201-1215. https://doi.org/10.1002/2016JF004027

Hibert, C., Malet, J., Bourrier, F., Provost, F., Berger, F., Bornemann, P., \& et al. (2017). Single-block rockfall dynamics inferred from seismic signal analysis. Earth Surface Dynamics, 5, 283-292. https://doi.org/10.5194/esurf-5-283-2017

Hibert, C., Mangeney, A., Grandjean, G., Baillard, C., Rivet, D., Shapiro, N. M., \& et al. (2014). Automated identification, location, and volume estimation of rockfalls at Piton de la Fournaise volcano. Journal of Geophysical Research: Earth Surface, 119, $1082-1105$. https://doi.org/10.1002/2013JF002970

Hibert, C., Mangeney, A., Grandjean, G., Peltier, A., DiMuro, A., Shapiro, N., \& et al. (2017). Spatio-temporal evolution of rockfall activity from 2007 to 2011 at the Piton de la Fournaise volcano inferred from seismic data. Journal of Volcanology and Geothermal Research, 333-334, 36-52. https://doi.org/10.1016/j.jvolgeores.2017.01.007

Hibert, C., Mangeney, A., Grandjean, G., \& Shapiro, N. M. (2011). Slope instabilities in Dolomieu crater, Réunion Island: From seismic signals to rockfall characteristics. Journal of Geophysical Research, 116, F04032. https://doi.org/10.1029/2011JF002038

Hogg, A. J. (2007). Two-dimensional granular slumps down slopes. Physics of Fluids, 19, 093301. https://doi.org/10.1063/1.2762254

Hsu, L., Dietrich, W. E., \& Sklar, L. S. (2014). Mean and fluctuating basal forces generated by granular flows: Laboratory observations in a large vertically rotating drum. Journal of Geophysical Research: Earth Surface, 119, 1283-1309. https://doi.org/10.1002/2013JF003078

Huang, C.-J., Shieh, C.-L., \& Yin, H.-Y. (2004). Laboratory study of the underground sound generated by debris flows. Journal of Geophysical Research, 109, F01008. https://doi.org/10.1029/2003JF000048

Huang, C.-J., Yin, H.-Y., Chen, C.-Y., Yeh, C.-H., \& Wang, C.-L. (2007). Ground vibrations produced by rock motions and debris flows. Journal of Geophysical Research, 112, F02014. https://doi.org/10.1029/2005JF000437

Iverson, R. (1997). The physics of debris flows. Reviews of Geophysics, 35, 245-296. https://doi.org/10.1029/97RG00426

Jessop, D. E., Kelfoun, K., Labazuy, P., Mangeney, A., \& Roche, O. (2012). LiDAR derived morphology of the 1993 lascar pyroclastic flow deposits, and implication for flow dynamics and rheology. Journal of Volcanology and Geothermal Research, 245-246, 81-97. https://doi.org/10.1016/j.jvolgeores.2012.06.030

Johnson, K. (1985). Contact mechanics. Cambridge, UK: Cambridge University Press.

Kanamori, H., \& Given, J. W. (1982). Analysis of long-period seismic waves excited by the May 18, 1980, eruption of Mount St. Helens-A terrestrial monopole. Journal of Geophysical Research, 87, 5422-5432. https://doi.org/10.1029/JB087iB07p05422

Kean, J., Coe, J., Coviello, V., Smith, J., McCoy, S., \& Arattano, M. (2015). Estimating rates of debris flow entrainment from ground vibrations. Geophysical Research Letters, 42, 6365-6372. https://doi.org/10.1002/2015GL064811

Lacaze, L., \& Kerswell, R. R. (2009). Axisymmetric granular collapse: A transient 3D flow test of viscoplasticity. Physical Review Letters, 102(10) 108305. https://doi.org/10.1103/PhysRevLett.102.108305

Lajeunesse, E., Mangeney-Castelnau, A., \& Vilotte, J. P. (2004). Spreading of a granular mass on a horizontal plane. Physics of Fluids, 16(7), 2371-2381. https://doi.org/10.1063/1.1736611

Lancieri, M., Madariaga, R., \& Bonilla, F. (2012). Spectral scaling of the aftershocks of the Tocopilla 2007 earthquake in northern Chile. Geophysical Journal International, 189(1), 469-480. https://doi.org/10.1111/j.1365-246X. 2011.05327.x

Lévy, C., Mangeney, A., Bonilla, F., Hibert, C., Calder, E., Smith, P., \& Cole, P. (2015). Friction weakening in granular flows deduced from seismic records at the Souffrière Hills Volcano, Montserrat. Journal of Geophysical Research: Solid Earth, 120, 7536-7557. https://doi.org/10.1002/2015JB012151

Lube, G., Huppert, H. E., Sparks, R. S. J., \& Freundt, A. (2005). Collapses of two-dimensional granular columns. Physical Review E, 72(4), 041301. https://doi.org/10.1103/PhysRevE.72.041301

Lube, G., Huppert, H. E., Sparks, R. S. J., \& Freundt, A. (2011). Granular column collapses down rough, inclined channels. Journal of Fluid Mechanics, 675, 347-368. https://doi.org/10.1017/jfm.2011.21

Lube, G., Huppert, H. E., Sparks, R. S. J., \& Hallworth, M. A. (2004). Axisymmetric collapses of granular columns. Journal of Fluid Mechanics, 508(1), 175-199. https://doi.org/10.1017/S0022112004009036

Mangeney, A., Roche, O., Hungr, O., Mangold, N., Faccanoni, G., \& Lucas, A. (2010). Erosion and mobility in granular collapse over sloping beds. Journal of Geophysical Research, 115, F03040. https://doi.org/10.1029/2009JF001462

Mangeney-Castelnau, A. (2003). Numerical modeling of avalanches based on Saint Venant equations using a kinetic scheme. Journal of Geophysical Research, 108(B11), 2527. https://doi.org/10.1029/2002JB002024

Moretti, L., Allstadt, K., Mangeney, A., Capdeville, Y., Stutzmann, E., \& Bouchut, F. (2015). Numerical modeling of the Mount Meager landslide constrained by its force history derived from seismic data. Journal of Geophysical Research: Solid Earth, 120, 2579-2599. https://doi.org/10.1002/2014JB011426

Moretti, L., Mangeney, A., Capdeville, Y., Stutzmann, E., Huggel, C., Schneider, D., \& Bouchut, F. (2012). Numerical modeling of the Mount Steller landslide flow history and of the generated long period seismic waves. Geophysical Research Letters, 39, L16402. https://doi.org/10.1029/2012GL052511

Pérez-Guillén, C., Sovilla, B., Suriñach, E., Tapiac, M., \& Köhler, A. (2016). Deducing avalanche size and flow regimes from seismic measurements. Cold Regions Science and Technology, 121, 25-41. https://doi.org/10.1016/j.coldregions.2015.10.004

Roche, O., Attali, M., Mangeney, A., \& Lucas, A. (2011). On the run-out distance of geophysical gravitational flows: Insight from fluidized granular collapse experiments. Earth and Planetary Science Letters, 311(3-4), 375-385. https://doi.org/10.1016/j.epsl.2011.09.023

Roche, O., Montserrat, S., Niño, Y., \& Tamburrino, A. (2008). Experimental observations of water-like behavior of initially fluidized, dam break granular flows and their relevance for the propagation of ash-rich pyroclastic flows. Journal of Geophysical Research, $113, \mathrm{~B} 12203$. https://doi.org/10.1029/2008JB005664

Roche, O., Niño, Y., Mangeney, A., Brand, B., Pollock, N., \& Valentine, G. A. (2013). Dynamic pore-pressure variations induce substrate erosion by pyroclastic flows. Geology, 41(10), 1107-1110. https://doi.org/10.1130/G34668.1

Royer, D., \& Dieulesaint, E. (2000). Elastic waves in solids I: Free and guided propagation. Berlin, Germany: Springer-Verlag.

Schneider, D., Bartelt, P., Caplan-Auerbach, J., Christen, M., Huggel, C., \& McArdell, B. W. (2010). Insights into rock-ice avalanche dynamics by combined analysis of seismic recordings and a numerical avalanche model. Journal of Geophysical Research, 115, F04026. https://doi.org/10.1029/2010JF001734

Siavoshi, S., \& Kudrolli, A. (2005). Failure of a granular step. Physical Review E, 71(5), 051302. https://doi.org/10.1103/PhysRevE.71.051302

Sulpizio, R., Castioni, D., Rodriguez-Sedano, L., Sarocchi, D., \& Lucchi, F. (2016). The influence of slope-angle ratio on the dynamics of granular flows: Insights from laboratory experiments. Bulletin of Volcanology, 78(11), 77. https://doi.org/10.1007/s00445-016-1069-5

Suriñach, E., Vilajosana, I., Khazaradze, G., Biescas, B., Furdada, G., \& Vilaplana, J. M. (2005). Seismic detection and characterization of landslides and other mass movements. Natural Hazards and Earth System Sciences, 5(6), 791-798. https://doi.org/10.5194/nhess-5-791-2005

Tsai, V. C., Minchew, B., Lamb, M. P., \& Ampuero, J.-P. (2012). A physical model for seismic noise generation from sediment transport in rivers. Geophysical Research Letters, 39, L02404. https://doi.org/10.1029/2011GL050255 
Vilajosana, I., Suriñach, E., Abellan, A., Khazaradze, G., Garcia, D., \& Llosa, J. (2008). Rockfall induced seismic signals: Case study in Montserrat, Catalonia. Natural Hazards and Earth System Sciences, 8(4), 805-812. https://doi.org/10.5194/nhess-8-805-2008

Weaver, R. L. (1985). Diffuse elastic waves at a free surface. Journal of the Acoustical Society of America, 78(1), $131-136$. https://doi.org/10.1121/1.392576

Yamada, M., Kumagai, H., Matsushi, Y., \& Matsuzawa, T. (2013). Dynamic landslide processes revealed by broadband seismic records. Geophysical Research Letters, 40, 2998-3002. https://doi.org/10.1002/grl.50437

Yamada, M., Mangeney, A., Matsushi, Y., \& Moretti, L. (2016). Estimation of dynamic friction process of the Akatani landslide based on the waveform inversion and numerical simulation. Geophysical Journal International, 206, 1479-1486. https://doi.org/10.1093/gji/ggw216

Zhao, J., Moretti, L., Mangeney, A., Stutzmann, E., Kanamori, H., Capdeville, Y., \& et al. (2015). Model space exploration for determining landslide source history from long-period seismic data. Pure and Applied Geophysics, 172(2), 389-413. https://doi.org/10.1007/s00024-014-0852-5 